\title{
Do methane seeps support distinct macrofaunal assemblages? Observations on community structure and nutrition from the northern California slope and shelf
}

\author{
Lisa A. Levin ${ }^{1, *}$, David W. James ${ }^{1}$, Christopher M. Martin ${ }^{1}$, Anthony E. Rathburn ${ }^{1}$, \\ Leslie H. Harris ${ }^{2}$, Robert H. Michener ${ }^{3}$ \\ ${ }^{1}$ Marine Life Research Group, Scripps Institution of Oceanography, La Jolla, California 92093-0218, USA \\ ${ }^{2}$ Los Angeles County Museum of Natural History, 900 Exposition Boulevard, Los Angeles, California 90007, USA \\ ${ }^{3}$ Boston University Stable Isotope Laboratory, Department of Biology, 5 Cummington St., Boston, Massachusetts 02215, USA
}

\begin{abstract}
Although the conspicuous epifauna of reducing environments are known to exhibit strong morphological, physiological, and nutritional adaptations for life in these habitats, it is less clear whether infaunal organisms do so as well. We examined metazoan macrofauna from methane-seep sediments on the northern California slope (500 to $525 \mathrm{~m}$ depth) and from seep and non-seep sediments at 3 locations on the shelf ( 31 to $53 \mathrm{~m}$ depth) to determine whether the community structure and nutritional sources of seep infauna were distinct from those in non-seep, margin sediments. Seep macrofauna consisted mainly of normal slope and shelf species found in productive settings. Several macrofaunal taxa, such as Capitella sp., Diastylopsis dawsoni, and Synidotea angulata, exhibited a preference for seeps. Other taxa, such as the amphipods Rhepoxynius abronius and $R$. daboius, avoided seeps. Species richness of shelf macrofauna, evaluated by rarefaction and diversity indices $\left(H^{\prime}\right.$ and $\left.J^{\prime}\right)$, generally did not differ in seep and non-seep sediments. Similarly, stable isotopic composition $\left(\delta^{13} C, \delta^{15} N\right)$ of active seep and non-seep macrofauna did not differ at the 3 shelf sites. Stable isotopic analyses of calcareous material confirmed the presence of methane-influenced pore waters at the slope study site. At one slope clam bed, macrofaunal $\delta^{13} \mathrm{C}$ signatures were lower and $\delta^{15} \mathrm{~N}$ values were higher than at another clam bed, inactive slope sediments and shelf sites. However, only 1 of 14 macrofaunal taxa (a dorvilleid polychaete) exhibited isotopic evidence of chemosynthetic nutritional sources. At these sites, seep influence on the ecology of continental margin infauna appears spatially limited and relatively subtle. At their current level of activity, the northern California slope and shelf seeps appear to function as ephemeral, smallscale disturbances that are not sufficiently persistent to allow chemosynthesis-based trophic specialization by most infauna. Rather, we suggest that many of the infauna inhabiting these seep sediments are shelf and slope species preadapted to organic-rich, reducing environments.
\end{abstract}

KEY WORDS: Macrofauna $\cdot$ Macrobenthos $\cdot$ Methane cold seep $\cdot$ Sulfide $\cdot$ Stable isotope $\cdot \delta^{13} \mathrm{C} \cdot$ $\delta^{15} \mathrm{~N} \cdot$ Chemoautotrophy $\cdot$ Diversity $\cdot$ Eel River margin

Resale or republication not permitted without written consent of the publisher

\section{INTRODUCTION}

Seafloor sediments provide varied environments for sediment-dwelling macrofauna (infauna). Because the

*E-mail: llevin@ucsd.edu physical and chemical properties of the sediments can strongly influence resident animals (McCall \& Tevesz 1982), processes that change these properties can potentially exert strong influence on infaunal communities. One such process is the venting of methane, and consequent microbially mediated oxidation, which is 
coupled with sulfate reduction in anaerobic environments (Beauchamp et al. 1989, Masuzawa et al. 1992). Since discovery of the first methane seeps (Paull et al. 1984), seep-like environments have been found in varied settings, including subduction zones, transform faults, mass wasting sites (Hovland \& Judd 1988, Sibuet \& Olu 1998), pockmarks (Dando et al. 1991, 1994), and around dead marine mammals (Smith et al. 1998). These sites are characterized by the presence of reduced sulfides and methane, limited oxygen within sediments, high microbial biomass (Guezennec \& Fiala 1996), and often a characteristic epibiota that includes symbiont-bearing clams, vestimentiferan tube worms and sometimes mussels or gastropods (Hovland \& Judd 1988, Sibuet \& Olu 1998). During the past decade, a large number of sites venting cold, methane-rich fluids have been discovered along the eastern and western Pacific margins. Sites of fluid flow in near-surface Pacific sediments include the Nankai Trough and Japan Trench (Boulegue et al. 1987a,b, Dron et al. 1987, Sibuet et al. 1988, Gamo et al. 1992), Gulf of Alaska (Suess et al. 1998), Oregon subduction zone (Han \& Suess 1986, Kulm et al. 1986, Suess \& Whiticar 1989), northern California slope (Brewer et al. 1997, Yun et al. 1999), Monterey Bay (Barry et al. 1996, 1997), accretionary prisms off Costa Rica (Zuleger et al. 1995, 1996) and Barbados (Olu et al. 1996b, 1997), and the Peruvian margin (Olu et al. 1996a).

Most reports of seep fauna have focused on the larger, surface-dwelling organisms (megafauna) (reviewed in Sibuet \& Olu 1998) but there are several reports of smaller infauna (Table 1); only a few of these examined infaunal community structure. Megafauna and macrofauna of methane seeps and pockmarks have been studied most thoroughly in shallow waters of the Santa Barbara margin (Davis \& Spiess 1980, Montagna et al. 1989), the North Sea (Dando et al. 1991, 1994, Jones 1993, 1996) and the Skagerrak (Dando et al. 1994). General patterns emerging from these studies suggest that densities of macrofauna living in seep environments may be elevated or reduced relative to non-seep conditions. In all instances where this was examined, dominance was high and species diversity was relatively low, relative to surrounding sediments. Several megafaunal or meiofaunal taxa at seeps belong to genera or families known to bear symbionts. Examples include pogonophorans, thyasirid, lucinid and vesicomyid clams, and various gutless nematodes (Table 1).

Analyses of the natural, stable isotopic compositions of tissues $\left(\delta^{13} \mathrm{C}, \delta^{15} \mathrm{~N}, \delta^{34} \mathrm{~S}\right)$ have been used as a means to determine food sources in organisms inhabiting reducing environments (e.g., Paull et al. 1985, Brooks et al. 1987, Cary et al. 1989, Kennicutt et al. 1989, Schmaljohann et al. 1990, Conway et al. 1994). The stable isotopic compositions $\left(\delta^{13} \mathrm{C}\right.$ and $\left.\delta^{18} \mathrm{O}\right)$ of carbonate skeletal material from deep-sea organisms can also be indicative of ambient temperature and chemistry conditions (Van Dover \& Fry 1989, Sen Gupta \& Aharon 1994). Large depletions of $\delta^{13} \mathrm{C}$ ( -30 to $-70 \%$ ) in the tissues of organisms, such as vestimentiferans, bivalves and gastropods, have been proposed to indicate methanotrophy or sulfide oxidation by symbionts in seep and vent taxa (Paull et al. 1985, Brooks et al. 1987, Van Dover \& Fry 1989). However, comparable isotopic signatures may be acquired from consumption of free-living chemosynthetic bacteria (e.g., Beggiatoa or Thioploca), or by predation on animals with endosymbionts. Plankton detrital matter and terrestrially derived organic matter are other possible food sources for continental margin seep faunas.

This paper examines the metazoan macrofauna within shelf and slope seep sediments on the Eel River margin of northern California, USA, and for the shelf assemblages, draws comparisons with non-seep faunas. The following questions are addressed: (1) Do sites of methane seepage support macrofaunal assemblages distinct from those in non-seep sediments with respect to composition and diversity? and (2) Do methane seep macrofauna derive nutrition from different sources than non-seep macrofauna? Specifically, do seep infauna utilize chemosynthetically fixed organic matter? This latter question was addressed using $\delta^{13} \mathrm{C}$ and $\delta^{15} \mathrm{~N}$ analyses.

\section{METHODS}

Macrofauna were sampled from northern California methane seeps located on the continental slope at 500 to $525 \mathrm{~m}$ depth during October 1997 and on the shelf at 31 to $53 \mathrm{~m}$ depth during October 1998 (Fig. 1). This margin is a dynamic environment subject to extensive, rapid deposition of flood-derived terrestrial sediment, episodic seismic activity characteristic of convergent margins, intense, winter-storm-induced sediment resuspension, and migration of subsurface gases and pore fluids that alter surface morphology and redistribute sediments (Alexander \& Simoneau 1999, Yun et al. 1999). Seep samples from the slope site were collected in 5 distinct Calyptogena pacifica clam beds $\left(40^{\circ} 47.08^{\prime} \mathrm{N}, 124^{\circ} 35.68^{\prime} \mathrm{W}\right)$ and nearby inactive areas $\left(40^{\circ} 47.12^{\prime} \mathrm{N}, 124^{\circ} 35.61^{\prime} \mathrm{W}\right)$ with tube cores $(8.3 \mathrm{~cm}$ inner diameter, 10 to $15 \mathrm{~cm}$ deep) using the ROV 'Scorpio' and Sea Cliff submersible. Macrofauna were examined from 6 clam bed cores, and from 2 cores in sediments away from clam beds (Table 2). Seep and non-seep sediments were sampled at 3 shelf sites: 1 north of the Eureka Harbor (Site A, 50 to $53 \mathrm{~m}$ deep, $40^{\circ} 49.87^{\prime} \mathrm{N}, 124^{\circ} 15.95^{\prime} \mathrm{W}$ ) and 2 south of the Harbor 
Table 1. Summary of infaunal studies (megafauna, macrofauna and meiofauna) in seep environments. PAH: polyaromatic hydrocarbon

\begin{tabular}{|c|c|c|c|c|c|}
\hline Location & Depth (m) & Seep type & $\begin{array}{l}\text { Major epifaunal } \\
\text { feature }\end{array}$ & Faunal observations & Source \\
\hline $\begin{array}{l}\text { Santa Barbara, } \\
\text { USA }\end{array}$ & 16 & Hydrocarbon & Beggiatoa mats & $\begin{array}{l}72 \% \text { of species and } 90 \% \text { of individuals } \\
\text { shared with non-seep sites } \\
60 \% \text { of spp. had higher densities } \\
\text { at seeps, especially tubificid } \\
\text { oligochaetes } \\
\text { Dominance and diversity similar } \\
\text { to outside seeps } \\
\text { More deposit feeders, fewer } \\
\text { carnivores at seeps } \\
\text { Nematodes dominate seep } \\
\text { meiofauna ( } 85 \%)\end{array}$ & $\begin{array}{l}\text { Davis \& Spies (1980) } \\
\text { Montagna et al. } \\
\text { (1989) }\end{array}$ \\
\hline $\begin{array}{l}\text { Flower Garden, } \\
\text { Gulf of Mexico, } \\
\text { USA }\end{array}$ & 70 & Brine, sulfides & Beggiatoa mats & $\begin{array}{l}\text { Meiofauna dominated by } \\
\text { gnathostomulida, platyhelminthes, } \\
\text { aschelmenthes, and amphipods } \\
\text { Fauna distinct from non-seep sites, } \\
\text { but densities similar } \\
\text { Nematodes show higher dominance, } \\
\text { body elongation }\end{array}$ & $\begin{array}{l}\text { Powell \& Bright } \\
\text { (1981) } \\
\text { Powell et al. (1983) } \\
\text { Jensen (1986) }\end{array}$ \\
\hline $\begin{array}{l}\text { Gulf of Mexico, } \\
\text { USA }\end{array}$ & $350-2200$ & $\begin{array}{l}\text { Hydrocarbon/ } \\
\text { methane } \\
\text { Ammonia }\end{array}$ & $\begin{array}{l}\text { Microbial mats, } \\
\text { tube worms, } \\
\text { clams, mussels }\end{array}$ & $\begin{array}{l}\text { Lucinid, thyasirid clams associated } \\
\text { with lithified sediments to } 65 \mathrm{~cm} \\
\text { deep. PAH present in tissues } \\
\delta^{13} \mathrm{C} \text { varies - multiple C sources }\end{array}$ & $\begin{array}{l}\text { MacDonald et al. } \\
\text { (1990) } \\
\text { Wade et al. (1989) } \\
\text { Brooks et al. (1987) }\end{array}$ \\
\hline Barbados Prism & $1000-2000$ & Methane seeps & & $\begin{array}{l}\text { Maldanidae, Echiura, Sipuncula } \\
\text { Polyplacophora }\end{array}$ & Olu et al. (1996b) \\
\hline Barbados Prism & 6800 & Methane seeps & & $\begin{array}{l}\text { Maldanidae, Chaetopteridae, } \\
\text { Ampharetidae, meiofauna } \\
\text { up to } 11000 \text { ind. } 10 \mathrm{~cm}^{-2}\end{array}$ & Olu et al. (1997) \\
\hline $\begin{array}{l}\text { Guaymas Basin, } \\
\text { Gulf of California, } \\
\text { Mexico }\end{array}$ & 2020 & $\begin{array}{l}\text { Hydrocarbon/ } \\
\text { hydrothermal } \\
\text { venting (hot) }\end{array}$ & $\begin{array}{l}\text { Beggiatoa mats, } \\
\text { Calyptogena pacifica }\end{array}$ & $\begin{array}{l}\text { High densities, low spp. richness } \\
\text { Patchy faunal distributions } \\
\text { Dorvilleid polychaetes dominate } \\
\text { Also, ampharetid Amphisamytha, } \\
\text { trochid gastropods, Exallopus, } \\
\text { Nuculana }\end{array}$ & Grassle et al. (1985) \\
\hline $\begin{array}{l}\text { Florida } \\
\text { Escarpment, } \\
\text { USA }\end{array}$ & 3300 & $\begin{array}{l}\text { Brine, methane, } \\
\text { sulfides }\end{array}$ & $\begin{array}{l}\text { Beggiatoa, tube } \\
\text { worms, mussels, } \\
\text { gastropods }\end{array}$ & $\begin{array}{l}3 \text { cores taken-some species also } \\
\text { present in Pacific vents } \\
\text { (Amphisamytha, Nicomache, } \\
\text { Hesiospira) }\end{array}$ & $\begin{array}{l}\text { Petrecca \& Grassle } \\
\text { (1987) } \\
\text { Cary et al. (1989) } \\
\text { Paul et al. (1984) }\end{array}$ \\
\hline $\begin{array}{l}\text { Hatsuchima Seep, } \\
\text { Japan }\end{array}$ & 1160 & Methane seep & Calyptogena soyae & $\begin{array}{l}\text { Meiofauna abundance at seep } \\
\text { similar to control } \\
\text { Nematodes less diverse at seep } \\
\text { Maldanids Nicomache present } \\
\text { N. ohta has Beggiatoa-like filaments }\end{array}$ & $\begin{array}{l}\text { Shirayama \& Ohta } \\
(1990) \\
\text { Miura \& Hashimoto } \\
(1991)\end{array}$ \\
\hline $\begin{array}{l}\text { Nankai Trough, } \\
\text { Japan Trench, }\end{array}$ & $\begin{array}{l}3830 \\
5900\end{array}$ & Methane seep & $\begin{array}{l}3 \text { spp. Calyptogena } \\
\text { C. phaseoliformis }\end{array}$ & $\begin{array}{l}\text { Serpulids associated with clams } \\
\text { Tubiculous polychaetes in } \\
\text { mudstone, caprellids }\end{array}$ & $\begin{array}{l}\text { Juniper \& Sibuet } \\
\text { (1987) }\end{array}$ \\
\hline $\begin{array}{l}\text { Kashima } \\
\text { Seamount, Japan }\end{array}$ & 5640 & & C. phaseoliformis & $\begin{array}{l}\text { Caprellid amphipods and tubiculous } \\
\text { polychaetes near clams }\end{array}$ & \\
\hline Laurentian Fan & 3850 & $\begin{array}{l}\text { Methane, } \\
\text { sulfides }\end{array}$ & Calyptogena & $\begin{array}{l}\text { Thyasira, Solemya, pogonophorans, } \\
\text { Spirobranchia, galatheid crabs }\end{array}$ & $\begin{array}{l}\text { Petrecca \& Grassle } \\
(1987)\end{array}$ \\
\hline North Sea & 115 & $\begin{array}{l}\text { Methane seep } \\
\text { Pockmarks }\end{array}$ & Beggiatoa & $\begin{array}{l}\text { Taxa with chemosynthetic } \\
\text { symbionts including Thyasira, } \\
\text { Astomonema, Siboglinium } \\
\text { Polychaetes rare, but many non-seep } \\
\text { taxa present } \\
\text { Macrofaunal dominance high, } \\
\text { diversity low } \\
\text { Nematodes dominated meiofauna }\end{array}$ & Dando et al. (1991) \\
\hline Kattegat, Denmark & 10 & $\begin{array}{l}\begin{array}{l}\text { Bubbling reefs } \\
\text { (carbonate) }\end{array} \\
\text { Sandy seeps }\end{array}$ & $\begin{array}{l}\text { Cancer pagurus } \\
\text { Homarus vulgaris } \\
\text { Metridium senile }\end{array}$ & $\begin{array}{l}\text { Reduced densities of nematodes, } \\
\text { oligochaetes and polychaetes } \\
\text { relative to nearby control sites } \\
\text { Infauna concentrated in upper } 2 \mathrm{~cm} \\
\text { Symbiont bearing nematodes to } 22 \mathrm{~cm} \\
\text { Leptonemella aphanothecae }\end{array}$ & \\
\hline $\begin{array}{l}\text { Skaggerak, } \\
\text { Denmark }\end{array}$ & 300 & Methane seep & & $\begin{array}{l}\text { Pogonophorans form } 63 \% \text { of } \\
\text { infauna. Also, Paramphinome, } \\
\text { Abra, Nucula, Amphiura }\end{array}$ & Dando et al. (1994) \\
\hline $\begin{array}{l}\text { Monterey Bay, } \\
\text { USA }\end{array}$ & 906 & Cold seep & $\begin{array}{l}\text { Beggiatoa, } \\
\text { Thioploca sp. mats } \\
\text { Calyptogena sp. }\end{array}$ & $\begin{array}{l}\text { Nematodes dominant } \\
\text { Nematode and ciliate biovolume } \\
\text { and density higher at seep than } \\
\text { control sites } \\
\text { Largest body diameter at seeps }\end{array}$ & Buck \& Barry (1998) \\
\hline
\end{tabular}




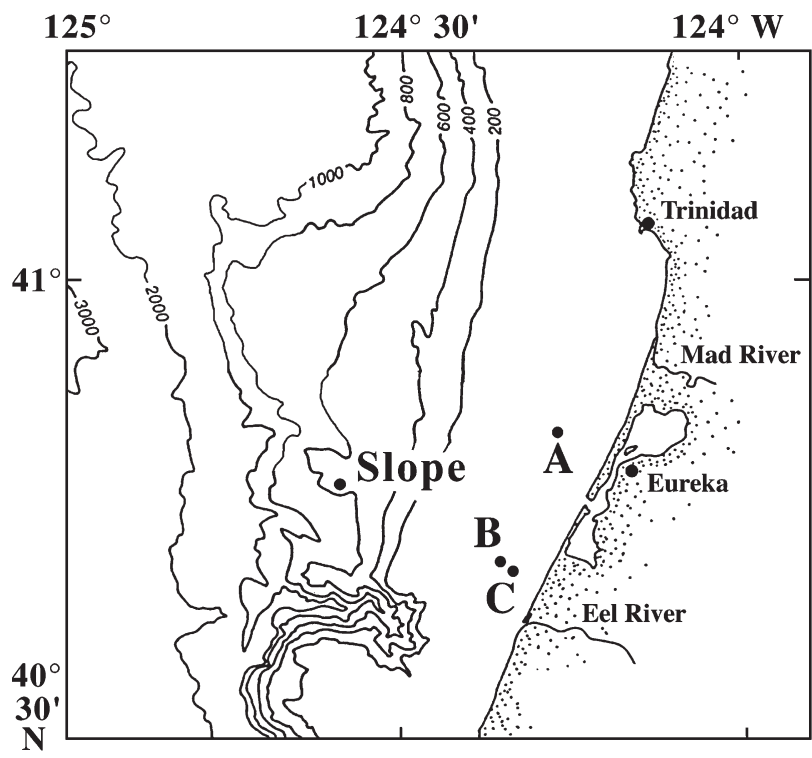

Fig. 1. Location of sampling stations on the northern California shelf and slope, USA

(Site B, 40 to $43 \mathrm{~m}$ deep, $40^{\circ} 42.22^{\prime} \mathrm{N}, 124^{\circ} 22.35^{\prime} \mathrm{W}$; Site C, 31 to $37 \mathrm{~m}$ deep, $\left.40^{\circ} 41.83^{\prime} \mathrm{N}, 124^{\circ} 21.37^{\prime} \mathrm{W}\right)$. Shelf sediments were sampled by the Delta submersible's slurp device and by shipek grabs deployed from the surface on the RV 'McGaw' (Table 2). The slurp and shipek samples collected the upper 6 to $8 \mathrm{~cm}$ of sediment, but were considered non-quantitative for analytical purposes.

On board ship, shipek samples were designated as seep samples if sediments were black and smelled sulfidic. They were considered non-seep samples if they were brown or tan and did not smell of sulfide. Five of the shipek samples appeared to be transitional between seep and non-seep sediments (gray sediments, slight sulfide smell). These were discarded from the analysis. Slurp samples were designated as seep samples if the scientist in the submersible viewed black surface sediments and methane bubbles emerging from the collection site.
The occurrence of methane seeps is well documented for the study sites (Brewer et al. 1997, Yun et al. 1999). They are oriented along deep-rooted structures in the accretionary wedge (e.g., faulted anticlines) (K. Brown pers. comm.). Although modern wood debris is ubiquitous in near-surface sediments on the Eel River margin, it is refractory. Degradation of ancient terrestrial detritus is the likely methane source on the shelf, while methane hydrates are the probable source of the observed sulfidic sediments and bubbles on the slope (Brewer et al. 1997).

On board ship, macrofauna samples were sieved on a $0.3 \mathrm{~mm}$ mesh and the material retained was preserved in $8 \%$ buffered formaldehyde and seawater. In the laboratory, macrofauna were sorted from sediments, counted, and identified to the lowest taxon possible. Macrofaunal diversity was examined using rarefaction analyses (Hurlbert 1971), the information index $\left(H^{\prime}\right.$ $\left.\log _{2}\right)$, and evenness $\left(J^{\prime}\right)$ with the software Biodiversity Pro (McAleece et al. 1997). Species count data were pooled within each station and treatment for the rarefaction analyses. Differences among sites and between seep versus non-seep sediments were examined for species composition and isotopic signatures with 1-way ANOVA or with $t$-tests using $\mathrm{JMP}^{\circledR}$ software. Proportions were arcsine-transformed prior to analysis. Multivariate analyses of community composition were performed by multidimensional scaling (MDS) and analysis of similarities (ANOSIM) (on uniform numbers of replicates) using Primer software (Clarke \& Warwick 1994). Multivariate analyses were conducted on species proportions because the shipek and slurp samples did not allow accurate density estimates.

At both shelf and slope sites, some macrofauna were sorted live on board ship, counted, identified and frozen in liquid nitrogen for subsequent analyses of $\delta^{13} \mathrm{C}$ and $\delta^{15} \mathrm{~N}$ (Table 2). Isotopic analyses were conducted using a Finnigan Conflo II continuous flow system and a Fisons NA1500 elemental analyzer. Prior to combustion, all samples were acidified with $1 \%$ $\mathrm{PtCl}_{2}$ to remove carbonates.

Surface sediments from the slope and each shelf site ( 0 to $2 \mathrm{~cm}$ deep) were frozen on board ship for later

Table 2. Number of samples examined for macrofauna on the northern California shelf and slope and number of individuals assayed for $\delta^{13} \mathrm{C}$ and $\delta^{15} \mathrm{~N}$

\begin{tabular}{|c|c|c|c|c|c|c|c|c|}
\hline & \multicolumn{2}{|r|}{ Slurp } & \multicolumn{2}{|c|}{ Shipek grab } & \multicolumn{2}{|c|}{ Tube cores } & \multicolumn{2}{|c|}{$\begin{array}{l}\text { Isotopic analyses } \\
\text { (no. of ind.) }\end{array}$} \\
\hline & Seep & Non-seep & Seep & Non-seep & Seep & Inactive & Seep & Non-seep \\
\hline Site A (50 m deep) & 5 & 2 & 3 & 3 & 0 & 0 & 20 & 11 \\
\hline Site B (40 m deep) & 4 & 4 & 6 & 6 & 0 & 0 & 2 & 2 \\
\hline Site C (35 m deep) & 0 & 2 & 6 & 33 & 0 & 0 & 19 & 15 \\
\hline Slope (500 m deep) & 0 & 0 & 0 & 0 & 6 & 2 & 19 & 7 \\
\hline
\end{tabular}


analyses of sand, carbonate, and organic carbon and nitrogen content. Percent sand $(>63 \mu \mathrm{m})$ and silt and clay $(<63 \mu \mathrm{m})$ were determined by wet sieving homogenized sediments through a $63 \mu \mathrm{m}$ screen, drying the sediments at $80^{\circ} \mathrm{C}$ for $48 \mathrm{~h}$, and weighing the fractions. Organic $\mathrm{C}$ and $\mathrm{N}$ contents were analyzed on freeze dried sediments using a Perkin-Elmer CHN analyzer after removing inorganic carbonate through dissolution in $10 \% \mathrm{HCl}$. Bottom water was collected in a Niskin bottle by the ROV Scorpio or Delta submersible from within $2 \mathrm{~m}$ of the sediment surface for oxygen analysis. Samples were preserved in volumetric flasks with $\mathrm{MnCl}_{2}$ and $\mathrm{NaOH} / \mathrm{NaI}$ at sea and returned to the Scripps Institution of Oceanography for analysis of oxygen concentration on a modified Technicon AA-II continuous flow analyzer.

\section{RESULTS}

\section{Site descriptions}

Active venting of methane bubbles, visible at the sea surface and on the seabed, was observed in the 3 shelf areas sampled. Methane seepage was widespread but patchy, with discoloration of sediments (indicating sulfidic patches) on scales of decimeters to a meter. Storms during the cruise produced bottom disturbances and bedload transport over the entire study area, preventing sampling by the Delta submersible on some days. No microbial mats or typical seep epifauna were evident. Sediments often were rippled and swell troughs were present. No carbonate concretions were visible on the surface. Epifaunal flatfish, decapods, and cnidarians were abundant at Site A. Mysids, isopods and small flatfish were visible at Sites B and C.

On the slope, dense aggregations of the clam Calyptogena pacifica were observed in beds ca 15 to $100 \mathrm{~cm}^{2}$, often with a mix of living and dead shells. The distribution of clam beds suggested that seepage occurred in patches over at least $1 \mathrm{~km}^{2}$ of seafloor. Sea pens, the sea star Rathbunaster californicus, and the cnidarian Anthomastus ritteri were common throughout the area, though the cnidarians were absent in clam beds. Although sediments contained fine mud and did not appear to be current swept, tidal forcing generated strong, periodic bottom currents. Bouldersized outcrops of authigenic carbonate were observed, with smaller carbonate nodules present within sediments. Rapid venting of methane bubbles was observed in a $2 \mathrm{~m}$ diameter depression at ca $520 \mathrm{~m}$. Venting of seep fluids is indicated by pore-water profiles in 10 to $23 \mathrm{~cm}$ deep cores from 3 slope clam beds (clam beds 2, 3 and 4). These profiles revealed upward advection of slightly more saline, and $\mathrm{Ca}$ - and $\mathrm{Mg}$ - depleted fluids (J. Gieskes unpubl. data). However, compositional changes were relatively small, indicating that upward moving fluids mixed substantially with seawater prior to reaching the sediment-water interface.

\section{Bottom-water and sediment properties}

Bottom-water oxygen concentrations were higher on the shelf $\left(2.74\right.$ to $\left.2.95 \mathrm{ml} \mathrm{l}^{-1}\right)$ than at the slope site $\left(0.76 \pm 0.02 \mathrm{ml} \mathrm{l}^{-1}\right)$, which was at the upper boundary of the oxygen minimum zone (Table 3 ). Sediments at all sites were relatively coarse grained, but were markedly sandier at the 2 shallowest sites (B and $\mathrm{C}_{i}$ 84 to $95 \%$ sand), than at Site A (63\% sand) or the slope site (69\% sand) (Table 3). Correspondingly, sediment organic C content was low ( $<1 \%$ ) at all sites, but was slightly higher in the finer-grained areas (Table 3). No marked differences in sand content or organic matter content were evident between seep and non-seep sediments within a site.

\section{Macrofaunal composition}

\section{Shelf settings}

A total of 1269, 892, and 925 infaunal animals (excluding mysids) were examined from shelf Sites A, B, and $\mathrm{C}$, respectively. Most of the macrofaunal species collected from the shelf seeps also were present in nearby non-seep sediments (Table 4). The assemblage collected at both seep and non-seep sites was typical for sandy shelf environments. Dominant taxa included the isopod Tecticeps convexus, the amphipod Rhepoxynius daboius, the cumacean Diastylopsis dawsoni, the gastropod Astyris gausapata, and the polychaetes Nephtys californiensis, N. cornuta, Lumbrineris sp., Amaeana occidentalis, Scoletoma tetraura, Magelona sacculata, and Mediomastus spp. (Table 4).

Of the 201 taxa observed on the shelf, 55 were found exclusively in seep patches and 59 were found exclusively in non-seep sites (Table 4). Seven taxa were found exclusively in seep sediments at 2 of the 3 shelf sites: Atylus tridens, Cheirimedeia zotea, Lampropidae, Photis brevipes, Spiochaetopterus costarum, Synidotea angulata, and Tubulanus polymorphus. Many of these were single collections from a site, however, and probably do not reflect real habitat preferences. Cheirimedeia zotea $\left(t_{74}=1.97, \mathrm{p}=0.053\right)$ and Synidotea angulata $\left(t_{74}=2.44, \mathrm{p}=0.017\right)$, however, exhibited significant seep preference. Capitella sp. was the only taxon found exclusively in seep sediments at each of the 3 shelf sites, and was absent in 
Table 3. Bottom-water oxygen and sediment properties on the northern California margin. $\mathrm{n}=1$ where no standard error (SE) is given

\begin{tabular}{|c|c|c|c|c|c|c|c|c|}
\hline & & \multirow{2}{*}{$\begin{array}{l}\text { Bottom-water dissolved } \\
\text { oxygen }\left(\mathrm{ml} \mathrm{l}^{-1}\right)\end{array}$} & \multicolumn{2}{|c|}{ Grain size (\%) } & \multirow{2}{*}{$\begin{array}{c}\mathrm{CaCO}_{3} \\
(\%)\end{array}$} & \multirow{2}{*}{$\begin{array}{c}\text { Org C } \\
(\%)\end{array}$} & \multirow{2}{*}{$\begin{array}{c}\underset{(\%)}{\operatorname{Org} N} \\
.\end{array}$} & \multirow[t]{2}{*}{$\mathrm{C}: \mathrm{N}$} \\
\hline & & & Sand & Silt and clay & & & & \\
\hline Site A (50 m deep) & & 2.95 & & & & & & \\
\hline Seep & $\begin{array}{l}\text { Mean } \\
\text { SE } \\
\mathrm{n}\end{array}$ & & 62.5 & 37.5 & 10.6 & 0.76 & 0.05 & 15.2 \\
\hline Non-seep & $\begin{array}{l}\text { Mean } \\
\text { SE } \\
\mathrm{n}\end{array}$ & & - & - & - & - & - & - \\
\hline Site B (40 m deep) & & - & & & & & & \\
\hline Seep & $\begin{array}{l}\text { Mean } \\
\text { SE } \\
\mathrm{n}\end{array}$ & & 83.7 & 16.3 & $\begin{array}{c}8.4 \\
(2.3) \\
2\end{array}$ & $\begin{array}{c}0.37 \\
(0.02) \\
2\end{array}$ & $\begin{array}{c}0.02 \\
(0.02) \\
2\end{array}$ & 16.4 \\
\hline Non-seep & $\begin{array}{l}\text { Mean } \\
\text { SE } \\
\mathrm{n}\end{array}$ & & 93.2 & 6.8 & $\begin{array}{c}8.0 \\
(1.4) \\
3\end{array}$ & $\begin{array}{c}0.31 \\
(0.02) \\
3\end{array}$ & $\begin{array}{c}0.01 \\
(0.01) \\
3\end{array}$ & 30.5 \\
\hline Site C (35 m deep) & & 2.74 & & & & & & \\
\hline Seep & $\begin{array}{l}\text { Mean } \\
\text { SE } \\
\mathrm{n}\end{array}$ & & 94.9 & 5.1 & $\begin{array}{c}11.0 \\
(0.5) \\
4\end{array}$ & $\begin{array}{c}0.31 \\
(0.03) \\
4\end{array}$ & $\begin{array}{c}0.01 \\
(0.01) \\
4\end{array}$ & 22.7 \\
\hline Non-seep & $\begin{array}{l}\text { Mean } \\
\mathrm{SE} \\
\mathrm{n}\end{array}$ & & 89.2 & 10.8 & $\begin{array}{c}7.7 \\
(1.6) \\
3\end{array}$ & $\begin{array}{c}0.33 \\
(0.02) \\
3\end{array}$ & $\begin{array}{c}0.03 \\
(0.02) \\
3\end{array}$ & 10.4 \\
\hline \multicolumn{9}{|l|}{ Slope (500 m deep) } \\
\hline Seep & $\begin{array}{l}\text { Mean } \\
\text { SE } \\
\mathrm{n}\end{array}$ & $\begin{array}{c}0.76 \\
(0.02) \\
9\end{array}$ & $\begin{array}{c}59.9 \\
(3.0) \\
2\end{array}$ & $\begin{array}{c}40.1 \\
(3.0) \\
2\end{array}$ & $\begin{array}{l}12.4 \\
(0.7) \\
2\end{array}$ & $\begin{array}{c}0.97 \\
(0.00) \\
2\end{array}$ & $\begin{array}{c}0.06 \\
(0.01) \\
2\end{array}$ & 16.8 \\
\hline
\end{tabular}

non-seep sediments $\left(t_{74}=2.57, \mathrm{p}=0.012\right)$. The cumaceans Diastylopsis dawsoni $\left(t_{74}=3.45, \mathrm{p}<0.001\right)$ and other Lampropidae $\left(t_{74}=1.96, \mathrm{p}=0.054\right)$ were present in non-seep sediments but were proportionally better represented in seep settings. In addition, mysids were observed to concentrate over black patches emitting methane bubbles at Sites B and C. The mysids were abundant in slurp samples but rarely appeared in shipek grabs. Because they were demersal rather than infaunal, they were not included in the community analyses.

Although numerous species were collected in greater numbers outside of seeps, only the amphipods Rhepoxynius abronius $\left(t_{74}=2.77, \mathrm{p}=0.007\right.$ ) and $R$. daboius $\left(t_{74}=3.62, \mathrm{p}<0.001\right)$ were proportionally more abundant in non-seep than seep sediments.

Multidimensional scaling of macrofaunal assemblage data indicated significant compositional differences among the 3 shelf Sites A, B, and C, and between shelf and slope communities (Fig. 2) (ANOSIM all pairwise comparisons $\mathrm{p}<0.001)$. Within each shelf site, comparisons of seep and non-seep faunas by sampling gear type (slurp or shipek) revealed no differences (Fig. 3) (ANOSIM Site A: shipek $p=0.800$, slurp $\mathrm{p}=0.333 ;$ Site B: shipek $\mathrm{p}=0.383$, slurp $\mathrm{p}=0.314$; Site C: shipek $\mathrm{p}=0.412$ ).

\section{Slope settings}

Most of the infaunal species present in cores from 4 Calyptogena pacifica beds were characteristic of $\mathrm{Pa}-$ cific, North American continental margin sediments (Blake \& Scott 1997) (Table 5). Annelids were the dominant taxon, comprising about two-thirds of the seep infaunal collections. The most common species at both seep and non-seep sites were Mediomastus californiensis, Levinsenia gracilis, Nephtys cornuta, and various tanaids, amphipods, and ophiuroids. We observed potential differences in faunal abundance and biomass within and among clam beds (Table 6). The core from the most sulfidic sediments (clam bed 5) yielded infaunal densities 2.25 to 5 times higher than those in other clam patches or non-seep sediments (Table 6). Species or groups such as Ophryotrocha sp., oligochaetes, pyramidellid and other bivalves, amphipods and tanaids were more abundant or exclusively present in seep sediments, but the small number of inactive sediment sam- 
Table 4. Counts and proportions of infaunal species in seep and non-seep shelf sediments, northern California margin. See Table 2 for the sample types and numbers. Prop. = Proportion

\begin{tabular}{|c|c|c|c|c|c|c|c|c|c|c|c|c|}
\hline \multirow{3}{*}{ Species } & \multirow{2}{*}{\multicolumn{4}{|c|}{$\varlimsup_{\text {Non-seep }}$ Site A $\frac{}{\text { Seep }}$}} & \multirow{3}{*}{\multicolumn{4}{|c|}{ Non-seep }} & \multirow{3}{*}{\multicolumn{4}{|c|}{ 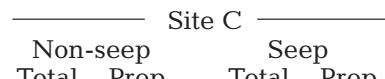 }} \\
\hline & & & & & & & & & & & & \\
\hline & Total & Prop. & Total & Prop. & & & & & & & & \\
\hline \multicolumn{13}{|l|}{ Cnidaria } \\
\hline Hydroid & 0 & 0.000 & 0 & 0.000 & 0 & 0.000 & 1 & 0.002 & 0 & 0.000 & 0 & 0.000 \\
\hline Obelia sp. & 0 & 0.000 & 2 & 0.002 & 0 & 0.000 & 0 & 0.000 & 0 & 0.000 & 0 & 0.000 \\
\hline Anemone & 0 & 0.000 & 0 & 0.000 & 1 & 0.002 & 1 & 0.002 & 6 & 0.007 & 0 & 0.000 \\
\hline Scolanthus sp. A & 0 & 0.000 & 1 & 0.001 & 0 & 0.000 & 0 & 0.000 & 0 & 0.000 & 0 & 0.000 \\
\hline Edwardsid? sp. & 3 & 0.010 & 0 & 0.000 & 0 & 0.000 & 0 & 0.000 & 0 & 0.000 & 0 & 0.000 \\
\hline \multicolumn{13}{|l|}{ Platyhelminthes } \\
\hline Turbellaria & 0 & 0.000 & 0 & 0.000 & 0 & 0.000 & 0 & 0.000 & 1 & 0.001 & 0 & 0.000 \\
\hline Polycladida & 0 & 0.000 & 1 & 0.001 & 0 & 0.000 & 0 & 0.000 & 0 & 0.000 & 0 & 0.000 \\
\hline Platyhelminthes sp. A & 0 & 0.000 & 1 & 0.001 & 0 & 0.000 & 0 & 0.000 & 1 & 0.001 & 0 & 0.000 \\
\hline \multicolumn{13}{|l|}{ Nemertea } \\
\hline Nemertea & 1 & 0.003 & 4 & 0.004 & 1 & 0.002 & 0 & 0.000 & 1 & 0.001 & 1 & 0.012 \\
\hline Cerebratulus sp. & 1 & 0.003 & 0 & 0.000 & 0 & 0.000 & 0 & 0.000 & 1 & 0.001 & 0 & 0.000 \\
\hline Lineus bilineatus & 0 & 0.000 & 1 & 0.001 & 0 & 0.000 & 0 & 0.000 & 0 & 0.000 & 0 & 0.000 \\
\hline Tubulanus polymorphus & 0 & 0.000 & 1 & 0.001 & 0 & 0.000 & 1 & 0.002 & 0 & 0.000 & 0 & 0.000 \\
\hline Annelida & & & & & & & & & & & & \\
\hline Amaeana occidentalis & 6 & 0.021 & 20 & 0.020 & 31 & 0.064 & 15 & 0.037 & 8 & 0.009 & 0 & 0.000 \\
\hline Ampharete arctica & 1 & 0.003 & 0 & 0.000 & 0 & 0.000 & 0 & 0.000 & 1 & 0.001 & 0 & 0.000 \\
\hline Ampharete finmarchica & 0 & 0.000 & 0 & 0.000 & 0 & 0.000 & 0 & 0.000 & 6 & 0.007 & 0 & 0.000 \\
\hline Ampharete labrops & 0 & 0.000 & 0 & 0.000 & 0 & 0.000 & 12 & 0.029 & 0 & 0.000 & 0 & 0.000 \\
\hline Ampharetidae juvenile & 0 & 0.000 & 16 & 0.016 & 1 & 0.002 & 0 & 0.000 & 0 & 0.000 & 0 & 0.000 \\
\hline Aphelochaeta sp. & 2 & 0.007 & 2 & 0.002 & 0 & 0.000 & 0 & 0.000 & 0 & 0.000 & 0 & 0.000 \\
\hline Aphrodita refulgida & 0 & 0.000 & 0 & 0.000 & 0 & 0.000 & 0 & 0.000 & 1 & 0.001 & 0 & 0.000 \\
\hline Apoprionospio pygmaea & 0 & 0.000 & 0 & 0.000 & 0 & 0.000 & 0 & 0.000 & 7 & 0.008 & 4 & 0.049 \\
\hline Aricidea (Acmira) catherinae & 1 & 0.003 & 4 & 0.004 & 0 & 0.000 & 1 & 0.002 & 2 & 0.002 & 0 & 0.000 \\
\hline Aricidea (Allia) antennata & 0 & 0.000 & 0 & 0.000 & 0 & 0.000 & 1 & 0.002 & 0 & 0.000 & 0 & 0.000 \\
\hline Aricidea pseudoarticulata & 0 & 0.000 & 0 & 0.000 & 0 & 0.000 & 0 & 0.000 & 1 & 0.001 & 0 & 0.000 \\
\hline Boccardia sp. & 0 & 0.000 & 0 & 0.000 & 1 & 0.002 & 0 & 0.000 & 0 & 0.000 & 0 & 0.000 \\
\hline Capitella sp. & 0 & 0.000 & 2 & 0.002 & 0 & 0.000 & 16 & 0.039 & 0 & 0.000 & 3 & 0.037 \\
\hline Capitellidae & 0 & 0.000 & 5 & 0.005 & 1 & 0.002 & 1 & 0.002 & 0 & 0.000 & 0 & 0.000 \\
\hline Chaetozone cf. hartmanae & 30 & 0.105 & 0 & 0.000 & 0 & 0.000 & 0 & 0.000 & 0 & 0.000 & 0 & 0.000 \\
\hline Chaetozone columbiana & 0 & 0.000 & 12 & 0.012 & 0 & 0.000 & 0 & 0.000 & 0 & 0.000 & 0 & 0.000 \\
\hline Chaetozone sp. & 0 & 0.000 & 11 & 0.011 & 0 & 0.000 & 0 & 0.000 & 1 & 0.001 & 0 & 0.000 \\
\hline Chaetozone sp. A & 4 & 0.014 & 7 & 0.007 & 0 & 0.000 & 1 & 0.002 & 20 & 0.024 & 2 & 0.025 \\
\hline Cirratulidae & 1 & 0.003 & 4 & 0.004 & 3 & 0.006 & 1 & 0.002 & 1 & 0.001 & 0 & 0.000 \\
\hline Cossura sp. & 1 & 0.003 & 1 & 0.001 & 0 & 0.000 & 0 & 0.000 & 0 & 0.000 & 0 & 0.000 \\
\hline Decamastus gracilis & 4 & 0.014 & 9 & 0.009 & 1 & 0.002 & 4 & 0.010 & 0 & 0.000 & 0 & 0.000 \\
\hline Dipolydora cardalia & 0 & 0.000 & 0 & 0.000 & 0 & 0.000 & 1 & 0.002 & 0 & 0.000 & 0 & 0.000 \\
\hline Dipolydora sp. juvenile & 0 & 0.000 & 1 & 0.001 & 0 & 0.000 & 0 & 0.000 & 0 & 0.000 & 0 & 0.000 \\
\hline Drilonereis sp. A & 6 & 0.021 & 0 & 0.000 & 0 & 0.000 & 0 & 0.000 & 0 & 0.000 & 0 & 0.000 \\
\hline Eteone cf. brigittae & 0 & 0.000 & 0 & 0.000 & 0 & 0.000 & 1 & 0.002 & 0 & 0.000 & 0 & 0.000 \\
\hline Eteone cf. spilotus & 3 & 0.010 & 0 & 0.000 & 1 & 0.002 & 0 & 0.000 & 1 & 0.001 & 0 & 0.000 \\
\hline Eteone sp. & 0 & 0.000 & 0 & 0.000 & 1 & 0.002 & 0 & 0.000 & 0 & 0.000 & 0 & 0.000 \\
\hline Eteone spilotus & 0 & 0.000 & 9 & 0.009 & 0 & 0.000 & 0 & 0.000 & 0 & 0.000 & 0 & 0.000 \\
\hline Euclymeninae & 0 & 0.000 & 1 & 0.001 & 0 & 0.000 & 0 & 0.000 & 0 & 0.000 & 0 & 0.000 \\
\hline Glycera cf. convoluta & 0 & 0.000 & 0 & 0.000 & 0 & 0.000 & 1 & 0.002 & 3 & 0.004 & 0 & 0.000 \\
\hline Glycera nana & 7 & 0.024 & 10 & 0.010 & 0 & 0.000 & 0 & 0.000 & 1 & 0.001 & 0 & 0.000 \\
\hline Glycera sp. A & 1 & 0.003 & 0 & 0.000 & 0 & 0.000 & 0 & 0.000 & 0 & 0.000 & 0 & 0.000 \\
\hline Glyceridae/Goniadidae & 0 & 0.000 & 1 & 0.001 & 0 & 0.000 & 0 & 0.000 & 0 & 0.000 & 0 & 0.000 \\
\hline Glycinde armigera & 1 & 0.003 & 4 & 0.004 & 1 & 0.002 & 0 & 0.000 & 2 & 0.002 & 0 & 0.000 \\
\hline Glycinde sp. & 1 & 0.003 & 2 & 0.002 & 0 & 0.000 & 0 & 0.000 & 0 & 0.000 & 0 & 0.000 \\
\hline Goniada maculata & 1 & 0.003 & 1 & 0.001 & 1 & 0.002 & 0 & 0.000 & 2 & 0.002 & 1 & 0.012 \\
\hline Goniada sp. & 1 & 0.003 & 0 & 0.000 & 0 & 0.000 & 0 & 0.000 & 0 & 0.000 & 0 & 0.000 \\
\hline Harmothoinae sp. A juvenile & 0 & 0.000 & 0 & 0.000 & 1 & 0.002 & 0 & 0.000 & 1 & 0.001 & 0 & 0.000 \\
\hline Hesionella sp. & 0 & 0.000 & 0 & 0.000 & 0 & 0.000 & 1 & 0.002 & 5 & 0.006 & 0 & 0.000 \\
\hline Hesionella sp. A & 0 & 0.000 & 1 & 0.001 & 0 & 0.000 & 0 & 0.000 & 2 & 0.002 & 0 & 0.000 \\
\hline Heteromastus filobranchus & 15 & 0.052 & 18 & 0.018 & 0 & 0.000 & 3 & 0.007 & 0 & 0.000 & 0 & 0.000 \\
\hline Heteromastus sp. & 2 & 0.007 & 1 & 0.001 & 0 & 0.000 & 0 & 0.000 & 0 & 0.000 & 0 & 0.000 \\
\hline Lanassa sp. juvenile & 0 & 0.000 & 1 & 0.001 & 0 & 0.000 & 0 & 0.000 & 0 & 0.000 & 0 & 0.000 \\
\hline Lanassa venusta & 0 & 0.000 & 1 & 0.001 & 0 & 0.000 & 0 & 0.000 & 0 & 0.000 & 0 & 0.000 \\
\hline Laonice cirrata & 4 & 0.014 & 4 & 0.004 & 0 & 0.000 & 0 & 0.000 & 0 & 0.000 & 0 & 0.000 \\
\hline Leitoscoloplos pugettensis & 9 & 0.031 & 56 & 0.057 & 15 & 0.031 & 14 & 0.034 & 31 & 0.037 & 1 & 0.012 \\
\hline Levinsenia gracilis & 1 & 0.003 & 1 & 0.001 & 0 & 0.000 & 0 & 0.000 & 0 & 0.000 & 0 & 0.000 \\
\hline Levinsenia oculata & 0 & 0.000 & 1 & 0.001 & 0 & 0.000 & 0 & 0.000 & 0 & 0.000 & 0 & 0.000 \\
\hline Levinsenia sp. juvenile & 0 & 0.000 & 2 & 0.002 & 0 & 0.000 & 0 & 0.000 & 0 & 0.000 & 0 & 0.000 \\
\hline Listriolobus sp. & 1 & 0.003 & 0 & 0.000 & 0 & 0.000 & 0 & 0.000 & 0 & 0.000 & 0 & 0.000 \\
\hline Lumbrineridae & 0 & 0.000 & 1 & 0.001 & 0 & 0.000 & 0 & 0.000 & 0 & 0.000 & 0 & 0.000 \\
\hline Lumbrineris californiensis & 0 & 0.000 & 0 & 0.000 & 1 & 0.002 & 0 & 0.000 & 6 & 0.007 & 0 & 0.000 \\
\hline Lumbrineris latreilli & 0 & 0.000 & 0 & 0.000 & 2 & 0.004 & 2 & 0.005 & 0 & 0.000 & 0 & 0.000 \\
\hline
\end{tabular}


Table 4 (continued)

\begin{tabular}{|c|c|c|c|c|c|c|c|c|c|c|c|c|}
\hline \multirow{3}{*}{ Species } & \multicolumn{4}{|c|}{- Site A $\longrightarrow$} & \multicolumn{4}{|c|}{ Site B } & \multicolumn{4}{|c|}{ Site C } \\
\hline & \multicolumn{2}{|c|}{ Non-seep } & \multicolumn{2}{|c|}{ Seep } & Non- & seep & $\mathrm{Se}$ & & Non & seep & Se & \\
\hline & Total & Prop. & Total & Prop. & Total & Prop. & Total & Prop. & Total & Prop. & Total & Prop. \\
\hline Lumbrineris sp. & 9 & 0.031 & 141 & 0.144 & 21 & 0.043 & 16 & 0.039 & 20 & 0.024 & 0 & 0.000 \\
\hline Magelona berkeleyi & 0 & 0.000 & 1 & 0.001 & 0 & 0.000 & 0 & 0.000 & 2 & 0.002 & 0 & 0.000 \\
\hline Magelona hartmanae & 0 & 0.000 & 0 & 0.000 & 0 & 0.000 & 0 & 0.000 & 7 & 0.008 & 0 & 0.000 \\
\hline Magelona sacculata & 0 & 0.000 & 0 & 0.000 & 8 & 0.017 & 10 & 0.025 & 68 & 0.081 & 11 & 0.136 \\
\hline Magelona sp. A & 0 & 0.000 & 1 & 0.001 & 0 & 0.000 & 0 & 0.000 & 0 & 0.000 & 0 & 0.000 \\
\hline Malmgreniella scriptoria & 0 & 0.000 & 0 & 0.000 & 25 & 0.052 & 9 & 0.022 & 1 & 0.001 & 0 & 0.000 \\
\hline Mediomastus ambisetus & 9 & 0.031 & 75 & 0.076 & 5 & 0.010 & 1 & 0.002 & 0 & 0.000 & 0 & 0.000 \\
\hline Mediomastus californiensis & 9 & 0.031 & 0 & 0.000 & 0 & 0.000 & 0 & 0.000 & 0 & 0.000 & 0 & 0.000 \\
\hline Mediomastus spp. & 0 & 0.000 & 102 & 0.104 & 15 & 0.031 & 2 & 0.005 & 0 & 0.000 & 1 & 0.012 \\
\hline Melina oculata & 0 & 0.000 & 1 & 0.001 & 0 & 0.000 & 0 & 0.000 & 0 & 0.000 & 0 & 0.000 \\
\hline Myriochele gracilis & 1 & 0.003 & 1 & 0.001 & 0 & 0.000 & 0 & 0.000 & 0 & 0.000 & 0 & 0.000 \\
\hline Myriochele oculata & 0 & 0.000 & 2 & 0.002 & 0 & 0.000 & 0 & 0.000 & 0 & 0.000 & 0 & 0.000 \\
\hline Myriochele sp. & 1 & 0.003 & 0 & 0.000 & 0 & 0.000 & 0 & 0.000 & 0 & 0.000 & 0 & 0.000 \\
\hline Myriochele sp. M & 1 & 0.003 & 0 & 0.000 & 0 & 0.000 & 0 & 0.000 & 0 & 0.000 & 0 & 0.000 \\
\hline Naineris? dendritica & 0 & 0.000 & 0 & 0.000 & 0 & 0.000 & 0 & 0.000 & 1 & 0.001 & 0 & 0.000 \\
\hline Nephtyidae & 0 & 0.000 & 1 & 0.001 & 0 & 0.000 & 1 & 0.002 & 10 & 0.012 & 0 & 0.000 \\
\hline Nephtys caecoides & 0 & 0.000 & 0 & 0.000 & 2 & 0.004 & 2 & 0.005 & 6 & 0.007 & 0 & 0.000 \\
\hline Nephtys californiensis & 4 & 0.014 & 11 & 0.011 & 48 & 0.099 & 16 & 0.039 & 158 & 0.187 & 15 & 0.185 \\
\hline Nephtys cornuta & 11 & 0.038 & 87 & 0.089 & 5 & 0.010 & 3 & 0.007 & 0 & 0.000 & 0 & 0.000 \\
\hline Nephtys ferruginea & 2 & 0.007 & 7 & 0.007 & 0 & 0.000 & 0 & 0.000 & 0 & 0.000 & 0 & 0.000 \\
\hline Nereididae & 0 & 0.000 & 0 & 0.000 & 1 & 0.002 & 0 & 0.000 & 0 & 0.000 & 0 & 0.000 \\
\hline Nereis sp. A & 0 & 0.000 & 0 & 0.000 & 0 & 0.000 & 2 & 0.005 & 0 & 0.000 & 0 & 0.000 \\
\hline Notomastus cf. lineatus & 0 & 0.000 & 0 & 0.000 & 9 & 0.019 & 2 & 0.005 & 3 & 0.004 & 2 & 0.025 \\
\hline Notomastus sp. & 0 & 0.000 & 3 & 0.003 & 5 & 0.010 & 0 & 0.000 & 0 & 0.000 & 0 & 0.000 \\
\hline Ophelina acuminata & 1 & 0.003 & 1 & 0.001 & 0 & 0.000 & 0 & 0.000 & 0 & 0.000 & 0 & 0.000 \\
\hline Orbinia johnsoni & 0 & 0.000 & 0 & 0.000 & 0 & 0.000 & 0 & 0.000 & 1 & 0.001 & 0 & 0.000 \\
\hline Orbiniidae & 1 & 0.003 & 3 & 0.003 & 1 & 0.002 & 1 & 0.002 & 2 & 0.002 & 0 & 0.000 \\
\hline Paraonidae & 0 & 0.000 & 6 & 0.006 & 0 & 0.000 & 2 & 0.005 & 3 & 0.004 & 0 & 0.000 \\
\hline Paraprionospio pinnata & 3 & 0.010 & 9 & 0.009 & 0 & 0.000 & 0 & 0.000 & 0 & 0.000 & 0 & 0.000 \\
\hline Pholoe glabra & 2 & 0.007 & 5 & 0.005 & 0 & 0.000 & 0 & 0.000 & 0 & 0.000 & 0 & 0.000 \\
\hline Phyllochaetopterus prolifica & 0 & 0.000 & 0 & 0.000 & 0 & 0.000 & 3 & 0.007 & 0 & 0.000 & 0 & 0.000 \\
\hline Phyllodoce longipes & 1 & 0.003 & 0 & 0.000 & 0 & 0.000 & 0 & 0.000 & 0 & 0.000 & 0 & 0.000 \\
\hline Phylo felix & 0 & 0.000 & 0 & 0.000 & 13 & 0.027 & 2 & 0.005 & 6 & 0.007 & 0 & 0.000 \\
\hline Podarkeopsis glabrus & 0 & 0.000 & 3 & 0.003 & 3 & 0.006 & 12 & 0.029 & 3 & 0.004 & 0 & 0.000 \\
\hline Polycirrus sp. & 0 & 0.000 & 0 & 0.000 & 0 & 0.000 & 0 & 0.000 & 0 & 0.000 & 1 & 0.012 \\
\hline Polynoidae & 0 & 0.000 & 5 & 0.005 & 0 & 0.000 & 0 & 0.000 & 0 & 0.000 & 0 & 0.000 \\
\hline Polynoidae sp. A & 0 & 0.000 & 0 & 0.000 & 1 & 0.002 & 0 & 0.000 & 0 & 0.000 & 0 & 0.000 \\
\hline Praxillella pacifica & 0 & 0.000 & 5 & 0.005 & 0 & 0.000 & 0 & 0.000 & 0 & 0.000 & 0 & 0.000 \\
\hline Prionospio (Minuspio) lighti & 3 & 0.010 & 14 & 0.014 & 1 & 0.002 & 1 & 0.002 & 3 & 0.004 & 0 & 0.000 \\
\hline Prionospio $(P$.$) steenstrupi$ & 3 & 0.010 & 2 & 0.002 & 0 & 0.000 & 0 & 0.000 & 1 & 0.001 & 0 & 0.000 \\
\hline Prionospio (P.) sp. A & 0 & 0.000 & 6 & 0.006 & 1 & 0.002 & 0 & 0.000 & 1 & 0.001 & 2 & 0.025 \\
\hline Prionospio spp. & 0 & 0.000 & 6 & 0.006 & 0 & 0.000 & 2 & 0.005 & 1 & 0.001 & 0 & 0.000 \\
\hline Proceraea sp. & 0 & 0.000 & 1 & 0.001 & 0 & 0.000 & 0 & 0.000 & 0 & 0.000 & 0 & 0.000 \\
\hline Scaleworm & 0 & 0.000 & 5 & 0.005 & 0 & 0.000 & 0 & 0.000 & 2 & 0.002 & 0 & 0.000 \\
\hline Scalibregma inflatum & 4 & 0.014 & 1 & 0.001 & 0 & 0.000 & 0 & 0.000 & 0 & 0.000 & 0 & 0.000 \\
\hline Scolelepis sp. & 0 & 0.000 & 0 & 0.000 & 0 & 0.000 & 0 & 0.000 & 2 & 0.002 & 0 & 0.000 \\
\hline Scoletoma tetraura & 20 & 0.070 & 19 & 0.019 & 19 & 0.039 & 5 & 0.012 & 22 & 0.026 & 1 & 0.012 \\
\hline Sigalion spinosus & 0 & 0.000 & 0 & 0.000 & 0 & 0.000 & 0 & 0.000 & 4 & 0.005 & 0 & 0.000 \\
\hline Sigalionidae & 0 & 0.000 & 0 & 0.000 & 2 & 0.004 & 0 & 0.000 & 6 & 0.007 & 0 & 0.000 \\
\hline Sphaerosyllis ranunculus & 0 & 0.000 & 5 & 0.005 & 0 & 0.000 & 0 & 0.000 & 0 & 0.000 & 0 & 0.000 \\
\hline Spiochaetopterus costarum & 0 & 0.000 & 1 & 0.001 & 0 & 0.000 & 1 & 0.002 & 0 & 0.000 & 0 & 0.000 \\
\hline Spionidae & 0 & 0.000 & 1 & 0.001 & 0 & 0.000 & 0 & 0.000 & 0 & 0.000 & 0 & 0.000 \\
\hline Spiophanes berkeleyorum & 3 & 0.010 & 11 & 0.011 & 2 & 0.004 & 1 & 0.002 & 2 & 0.002 & 1 & 0.012 \\
\hline Spiophanes bombyx & 0 & 0.000 & 0 & 0.000 & 1 & 0.002 & 0 & 0.000 & $2 \overline{6}$ & 0.031 & 5 & 0.062 \\
\hline Sternaspis fossor & 6 & 0.021 & 7 & 0.007 & 0 & 0.000 & 0 & 0.000 & 0 & 0.000 & 0 & 0.000 \\
\hline Streposyllis cf. minuta & 0 & 0.000 & 0 & 0.000 & 0 & 0.000 & 0 & 0.000 & 8 & 0.009 & 0 & 0.000 \\
\hline Trochochaeta multisetosa & 8 & 0.028 & 16 & 0.016 & 0 & 0.000 & 0 & 0.000 & 0 & 0.000 & 0 & 0.000 \\
\hline Mollusca & & & & & & & & & & & & \\
\hline Epitoniidae & 0 & 0.000 & 1 & 0.001 & 0 & 0.000 & 0 & 0.000 & 0 & 0.000 & 0 & 0.000 \\
\hline Astyris gausapata & 15 & 0.052 & 18 & 0.018 & 26 & 0.054 & 39 & 0.096 & 4 & 0.005 & 0 & 0.000 \\
\hline Nassarius mendicus & 0 & 0.000 & 11 & 0.011 & 0 & 0.000 & 1 & 0.002 & 1 & 0.001 & 0 & 0.000 \\
\hline Olivella pycna & 0 & 0.000 & 1 & 0.001 & 3 & 0.006 & 1 & 0.002 & 34 & 0.040 & 13 & 0.160 \\
\hline Odostomia sp. & 0 & 0.000 & 1 & 0.001 & 0 & 0.000 & 0 & 0.000 & 0 & 0.000 & 0 & 0.000 \\
\hline Turbonilla sp. & 0 & 0.000 & 2 & 0.002 & 0 & 0.000 & 0 & 0.000 & 0 & 0.000 & 0 & 0.000 \\
\hline Bivalve & 0 & 0.000 & 0 & 0.000 & 0 & 0.000 & 1 & 0.002 & 0 & 0.000 & 0 & 0.000 \\
\hline Axinopsida serricata & 1 & 0.003 & 8 & 0.008 & 1 & 0.002 & 2 & 0.005 & 1 & 0.001 & 0 & 0.000 \\
\hline Rochefortia tumida & 23 & 0.080 & 10 & 0.010 & 1 & 0.002 & 0 & 0.000 & 1 & 0.001 & 0 & 0.000 \\
\hline Macoma carlottensis & 0 & 0.000 & 0 & 0.000 & 1 & 0.002 & 0 & 0.000 & 3 & 0.004 & 0 & 0.000 \\
\hline Macoma nasuta & 0 & 0.000 & 2 & 0.002 & 0 & 0.000 & 0 & 0.000 & 4 & 0.005 & 0 & 0.000 \\
\hline Tellina modesta & 0 & 0.000 & 1 & 0.001 & 2 & 0.004 & 0 & 0.000 & 9 & 0.011 & 1 & 0.012 \\
\hline Gadila aberrans & 2 & 0.007 & 2 & 0.002 & 0 & 0.000 & 0 & 0.000 & 0 & 0.000 & 0 & 0.000 \\
\hline
\end{tabular}


Table 4 (continued)

\begin{tabular}{|c|c|c|c|c|c|c|c|c|c|c|c|c|}
\hline \multirow{3}{*}{ Species } & \multicolumn{4}{|c|}{$\longrightarrow$ Site A } & \multicolumn{4}{|c|}{ Site B } & \multicolumn{4}{|c|}{ Site C } \\
\hline & \multicolumn{2}{|c|}{ Non-seep } & \multicolumn{2}{|c|}{ Seep } & Non- & seep & $\mathrm{Se}$ & & Non- & seep & Se€ & \\
\hline & Total & Prop. & Total & Prop. & Total & Prop. & Total & Prop. & Total & Prop. & Total & Prop. \\
\hline Mytilidae & 0 & 0.000 & 1 & 0.001 & 0 & 0.000 & 0 & 0.000 & 0 & 0.000 & 0 & 0.000 \\
\hline Solemya reidi & 0 & 0.000 & 2 & 0.002 & 0 & 0.000 & 0 & 0.000 & 0 & 0.000 & 0 & 0.000 \\
\hline Yoldia seminuda & 1 & 0.003 & 0 & 0.000 & 0 & 0.000 & 0 & 0.000 & 0 & 0.000 & 0 & 0.000 \\
\hline Yoldia sp. & 0 & 0.000 & 1 & 0.001 & 0 & 0.000 & 0 & 0.000 & 0 & 0.000 & 0 & 0.000 \\
\hline Crustacea & & & & & & & & & & & & \\
\hline Ampelisca agassizi & 0 & 0.000 & 0 & 0.000 & 0 & 0.000 & 0 & 0.000 & 1 & 0.001 & 0 & 0.000 \\
\hline Ampelisca careyi & 1 & 0.003 & 3 & 0.003 & 10 & 0.021 & 3 & 0.007 & 32 & 0.038 & 1 & 0.012 \\
\hline Amphipod unidentified & 0 & 0.000 & 0 & 0.000 & 2 & 0.004 & 0 & 0.000 & 3 & 0.004 & 0 & 0.000 \\
\hline Argissa hamatipes & 0 & 0.000 & 1 & 0.001 & 0 & 0.000 & 0 & 0.000 & 0 & 0.000 & 0 & 0.000 \\
\hline Atylus tridens & 0 & 0.000 & 1 & 0.001 & 0 & 0.000 & 1 & 0.002 & 0 & 0.000 & 0 & 0.000 \\
\hline Cheirimedeia zotea & 0 & 0.000 & 3 & 0.003 & 0 & 0.000 & 3 & 0.007 & 0 & 0.000 & 0 & 0.000 \\
\hline Dyopedos sp. & 0 & 0.000 & 0 & 0.000 & 1 & 0.002 & 0 & 0.000 & 0 & 0.000 & 0 & 0.000 \\
\hline Eohaustorius sencillus & 0 & 0.000 & 0 & 0.000 & 0 & 0.000 & 0 & 0.000 & 2 & 0.002 & 0 & 0.000 \\
\hline Ericthonius brasiliensis & 0 & 0.000 & 0 & 0.000 & 8 & 0.017 & 17 & 0.042 & 0 & 0.000 & 0 & 0.000 \\
\hline Foxiphalus obtusidens & 0 & 0.000 & 0 & 0.000 & 0 & 0.000 & 0 & 0.000 & 5 & 0.006 & 0 & 0.000 \\
\hline Foxiphalus xiximeus & 0 & 0.000 & 0 & 0.000 & 25 & 0.052 & 0 & 0.000 & 0 & 0.000 & 0 & 0.000 \\
\hline Gammaridea & 0 & 0.000 & 0 & 0.000 & 0 & 0.000 & 0 & 0.000 & 3 & 0.004 & 0 & 0.000 \\
\hline Gammaropsis thompsoni & 0 & 0.000 & 5 & 0.005 & 3 & 0.006 & 9 & 0.022 & 0 & 0.000 & 0 & 0.000 \\
\hline Isaeidae & 0 & 0.000 & 0 & 0.000 & 0 & 0.000 & 2 & 0.005 & 0 & 0.000 & 0 & 0.000 \\
\hline Majoxiphalus major & 0 & 0.000 & 0 & 0.000 & 3 & 0.006 & 0 & 0.000 & 4 & 0.005 & 0 & 0.000 \\
\hline Microjassa bousfieldi & 0 & 0.000 & 0 & 0.000 & 1 & 0.002 & 0 & 0.000 & 0 & 0.000 & 0 & 0.000 \\
\hline Neoischyrocerus claustris & 0 & 0.000 & 0 & 0.000 & 0 & 0.000 & 1 & 0.002 & 0 & 0.000 & 0 & 0.000 \\
\hline Oedicerotidae & 0 & 0.000 & 0 & 0.000 & 0 & 0.000 & 2 & 0.005 & 0 & 0.000 & 0 & 0.000 \\
\hline Pachynus barnardi & 1 & 0.003 & 0 & 0.000 & 0 & 0.000 & 0 & 0.000 & 0 & 0.000 & 0 & 0.000 \\
\hline Pacifoculodes spinipes & 0 & 0.000 & 0 & 0.000 & 2 & 0.004 & 2 & 0.005 & 3 & 0.004 & 0 & 0.000 \\
\hline Photis brevipes & 0 & 0.000 & 1 & 0.001 & 0 & 0.000 & 1 & 0.002 & 0 & 0.000 & 0 & 0.000 \\
\hline Photis parvidons & 0 & 0.000 & 0 & 0.000 & 1 & 0.002 & 0 & 0.000 & 6 & 0.007 & 0 & 0.000 \\
\hline Photis sp. & 0 & 0.000 & 0 & 0.000 & 0 & 0.000 & 10 & 0.025 & 1 & 0.001 & 0 & 0.000 \\
\hline Phoxocephalidae & 1 & 0.003 & 5 & 0.005 & 16 & 0.033 & 2 & 0.005 & 31 & 0.037 & 5 & 0.062 \\
\hline Pleusymtes sp. & 0 & 0.000 & 0 & 0.000 & 0 & 0.000 & 2 & 0.005 & 0 & 0.000 & 0 & 0.000 \\
\hline Protomedeia prudens & 0 & 0.000 & 1 & 0.001 & 0 & 0.000 & 0 & 0.000 & 0 & 0.000 & 0 & 0.000 \\
\hline Protomedeia sp. juvenile & 1 & 0.003 & 0 & 0.000 & 0 & 0.000 & 0 & 0.000 & 0 & 0.000 & 0 & 0.000 \\
\hline Rhepoxynius abronius & 5 & 0.017 & 2 & 0.002 & 12 & 0.025 & 0 & 0.000 & 15 & 0.018 & 0 & 0.000 \\
\hline Rhepoxynius daboius & 2 & 0.007 & 3 & 0.003 & 19 & 0.039 & 1 & 0.002 & 59 & 0.070 & 0 & 0.000 \\
\hline Rhepoxynius homocuspidatus & 0 & 0.000 & 0 & 0.000 & 1 & 0.002 & 0 & 0.000 & 6 & 0.007 & 0 & 0.000 \\
\hline Rhepoxynius lucubrans & 0 & 0.000 & 0 & 0.000 & 1 & 0.002 & 0 & 0.000 & 0 & 0.000 & 0 & 0.000 \\
\hline Rhepoxynius stenodes & 0 & 0.000 & 1 & 0.001 & 0 & 0.000 & 3 & 0.007 & 4 & 0.005 & 0 & 0.000 \\
\hline Rhepoxynius vigitegus & 0 & 0.000 & 0 & 0.000 & 0 & 0.000 & 0 & 0.000 & 1 & 0.001 & 0 & 0.000 \\
\hline Synchelidium sp. & 0 & 0.000 & 0 & 0.000 & 3 & 0.006 & 1 & 0.002 & 2 & 0.002 & 0 & 0.000 \\
\hline Tiron biocellata & 0 & 0.000 & 7 & 0.007 & 1 & 0.002 & 5 & 0.012 & 0 & 0.000 & 0 & 0.000 \\
\hline Westwoodilla caecula & 2 & 0.007 & 0 & 0.000 & 0 & 0.000 & 0 & 0.000 & 0 & 0.000 & 0 & 0.000 \\
\hline Diastylis quadriplicata & 0 & 0.000 & 0 & 0.000 & 1 & 0.002 & 1 & 0.002 & 0 & 0.000 & 0 & 0.000 \\
\hline Diastylis santamariensis & 1 & 0.003 & 0 & 0.000 & 0 & 0.000 & 0 & 0.000 & 0 & 0.000 & 0 & 0.000 \\
\hline Diastylopsis dawsoni & 11 & 0.038 & 42 & 0.043 & 3 & 0.006 & 10 & 0.025 & 7 & 0.008 & 4 & 0.049 \\
\hline Eudorella pacifica & 3 & 0.010 & 2 & 0.002 & 0 & 0.000 & 0 & 0.000 & 0 & 0.000 & 0 & 0.000 \\
\hline Lampropidae & 0 & 0.000 & 2 & 0.002 & 0 & 0.000 & 1 & 0.002 & 0 & 0.000 & 0 & 0.000 \\
\hline Mesolamprops dillonensis & 0 & 0.000 & 0 & 0.000 & 1 & 0.002 & 0 & 0.000 & 0 & 0.000 & 0 & 0.000 \\
\hline Crangon alaskensis & 0 & 0.000 & 0 & 0.000 & 0 & 0.000 & 3 & 0.007 & 0 & 0.000 & 0 & 0.000 \\
\hline Eualus pusiolus & 0 & 0.000 & 1 & 0.001 & 1 & 0.002 & 1 & 0.002 & 0 & 0.000 & 0 & 0.000 \\
\hline Lissocrangon stylirostris & 0 & 0.000 & 0 & 0.000 & 1 & 0.002 & 0 & 0.000 & 2 & 0.002 & 0 & 0.000 \\
\hline Pagurus armatus & 0 & 0.000 & 0 & 0.000 & 2 & 0.004 & 0 & 0.000 & 0 & 0.000 & 0 & 0.000 \\
\hline Pinnixa franciscana & 1 & 0.003 & 0 & 0.000 & 0 & 0.000 & 0 & 0.000 & 0 & 0.000 & 0 & 0.000 \\
\hline Pinnixa occidentalis & 0 & 0.000 & 0 & 0.000 & 0 & 0.000 & 0 & 0.000 & 1 & 0.001 & 0 & 0.000 \\
\hline Edotia sublittoralis & 0 & 0.000 & 0 & 0.000 & 0 & 0.000 & 0 & 0.000 & 1 & 0.001 & 0 & 0.000 \\
\hline Isopod unidentified & 0 & 0.000 & 0 & 0.000 & 0 & 0.000 & 2 & 0.005 & 0 & 0.000 & 0 & 0.000 \\
\hline Munnogonium tillerae & 3 & 0.010 & 2 & 0.002 & 1 & 0.002 & 0 & 0.000 & 0 & 0.000 & 1 & 0.012 \\
\hline Pleurogonium californiense & 0 & 0.000 & 0 & 0.000 & 1 & 0.002 & 0 & 0.000 & 0 & 0.000 & 0 & 0.000 \\
\hline Synidotea angulata & 0 & 0.000 & 2 & 0.002 & 0 & 0.000 & 5 & 0.012 & 0 & 0.000 & 0 & 0.000 \\
\hline Synidotea sp. juvenile & 0 & 0.000 & 0 & 0.000 & 2 & 0.004 & 1 & 0.002 & 15 & 0.018 & 0 & 0.000 \\
\hline Tecticeps convexus & 2 & 0.007 & 19 & 0.019 & 71 & 0.147 & 98 & 0.240 & 67 & 0.079 & 3 & 0.037 \\
\hline Uromunna ubiquita & 0 & 0.000 & 0 & 0.000 & 0 & 0.000 & 3 & 0.007 & 0 & 0.000 & 0 & 0.000 \\
\hline Sipunculida & & & & & & & & & & & & \\
\hline Sipuncula juvenile & 0 & 0.000 & 1 & 0.001 & 0 & 0.000 & 0 & 0.000 & 1 & 0.001 & 0 & 0.000 \\
\hline Echinodermata & & & & & & & & & & & & \\
\hline Amphiodia urtica & 1 & 0.003 & 6 & 0.006 & 5 & 0.010 & 2 & 0.005 & 33 & 0.039 & 2 & 0.025 \\
\hline Lovenia cordiformis & 0 & 0.000 & 0 & 0.000 & 0 & 0.000 & 0 & 0.000 & 1 & 0.001 & 0 & 0.000 \\
\hline Hemichordata & & & & & & & & & & & & \\
\hline Enteropneusta & 0 & 0.000 & 0 & 0.000 & 1 & 0.002 & 0 & 0.000 & 0 & 0.000 & 0 & 0.000 \\
\hline Chaetognatha & & & & & & & & & & & & \\
\hline Chaetognath & 0 & 0.000 & 26 & 0.026 & 0 & 0.000 & 0 & 0.000 & 0 & 0.000 & 0 & 0.000 \\
\hline Total & 287 & & 982 & & 484 & & 408 & & 844 & & 81 & \\
\hline
\end{tabular}




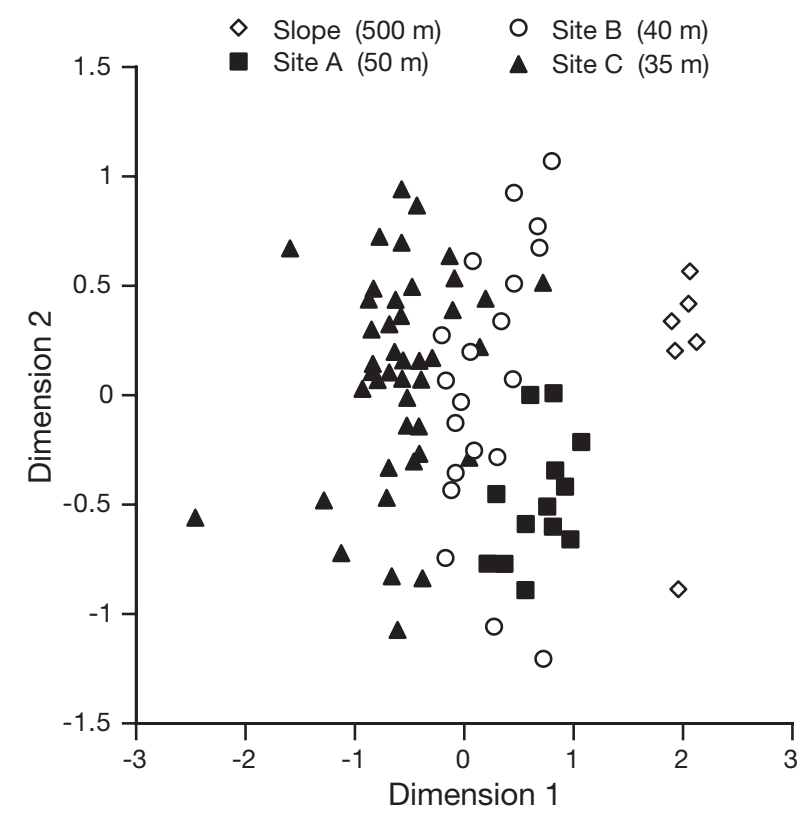

Fig. 2. Nondimensional MDS plot of macrofauna from sediments (seep and non-seep combined) at shelf Sites A, B, C, and from the slope on the northern California margin, USA. Stress value is 0.21

ples prevents conclusive statements about seep preference. A species accumulation curve for the slope macrofauna (Biodiversity Pro, 25 randomizations) suggests that 6 samples are insufficient to fully characterize the seep fauna.

\section{Macrofaunal diversity}

Rarefaction analyses, conducted to normalize species richness estimates for samples of different size, suggest there were few within-site differences in species richness between seep and non-seep sediments (Fig. 4). On average, diversity was relatively high at all sites. Information $\left(H^{\prime}\left[\log _{2}\right]\right.$, range 3.8 to 5.2$)$ and evenness indices $\left(J^{\prime}\right.$, range 0.76 to 0.87$)$, exhibited greater differences among shelf sites than between seep and non-seep sediments within a site (Table 7). Diversity was remarkably similar at all shelf and slope sites except in Site C seep sediments, where species richness appeared lower (Fig. 4, Table 7). Here 3 species, Magelona sacculata, Nephtys californiensis, and Olivella pycna, comprised $48 \%$ of the macrofauna.

\section{Macrofaunal nutrition}

Seep and non-seep infauna exhibited similar $\delta^{13} \mathrm{C}$ and $\delta^{15} \mathrm{~N}$ signatures within each of the 3 shelf stations (Table 8). Mean $\delta^{13} \mathrm{C}$ values ranged from -17 to -19 ,
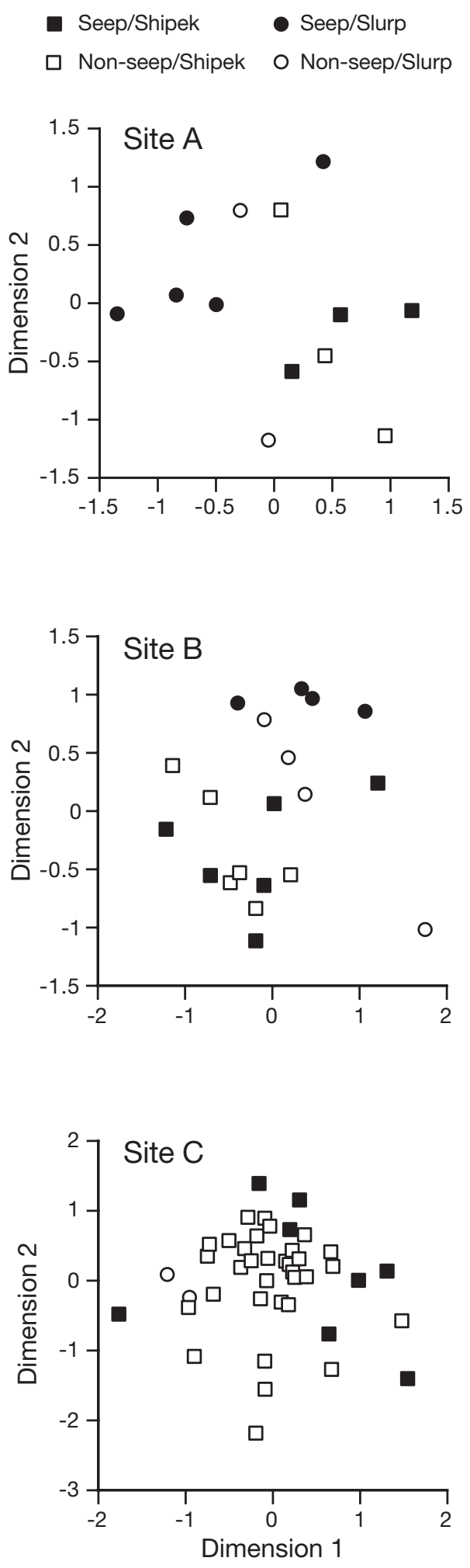

Fig. 3. Nondimensional MDS plot of macrofauna from seep and non-seep sediments at Sites A (50 m deep), B (40 m deep), and C (35 m deep) on the northern California shelf, USA. Samples taken by submersible suction (slurp) and shipek grab from the surface are indicated. Stress values are 0.14 for Site A, 0.14 for Site B, and 0.21 for Site C 
Table 5. Macrofaunal densities (per $54 \mathrm{~cm}^{2}$ core) in Calyptogena pacifica beds and inactive sediments on the Eel River margin slope

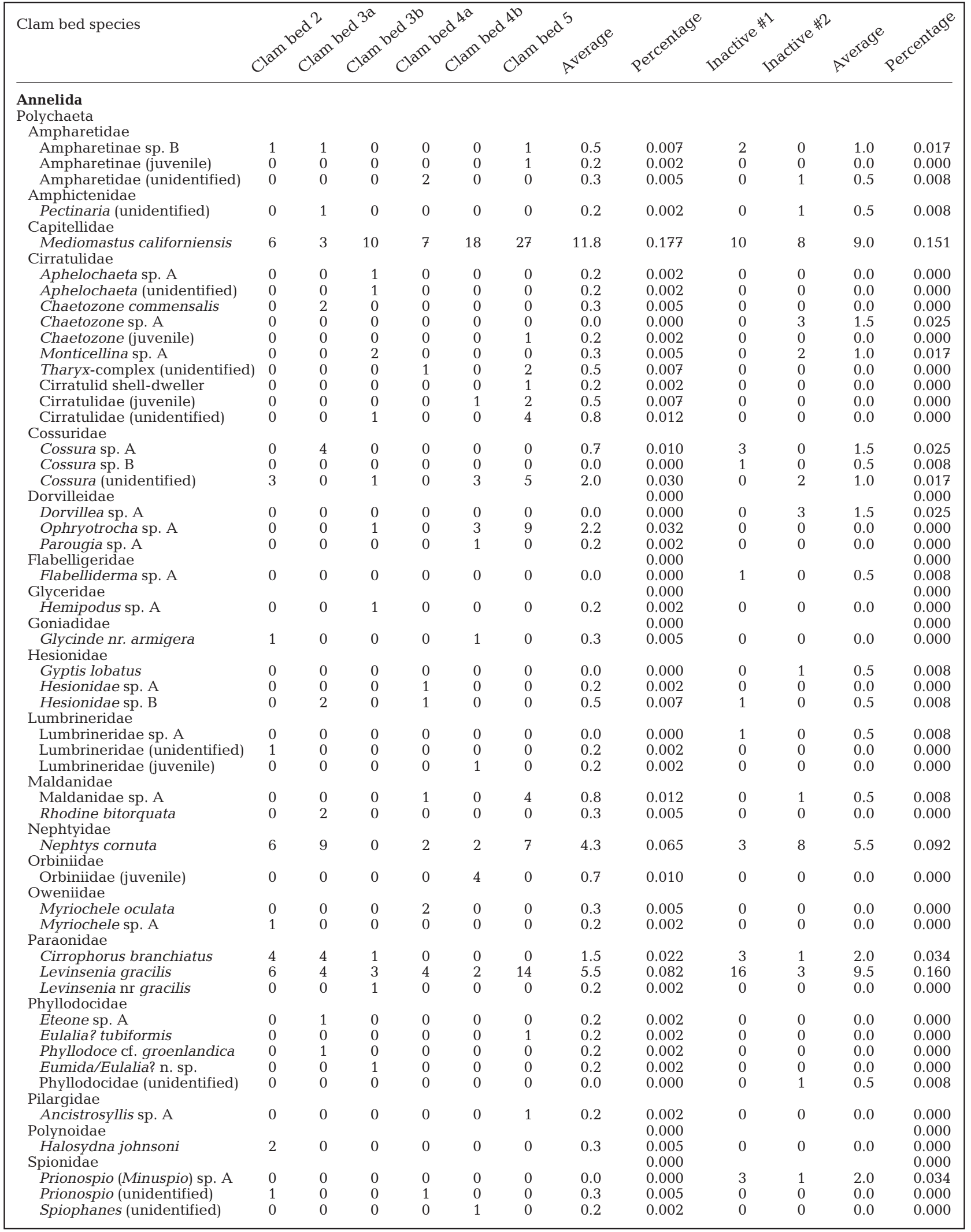


Table 5 (continued)

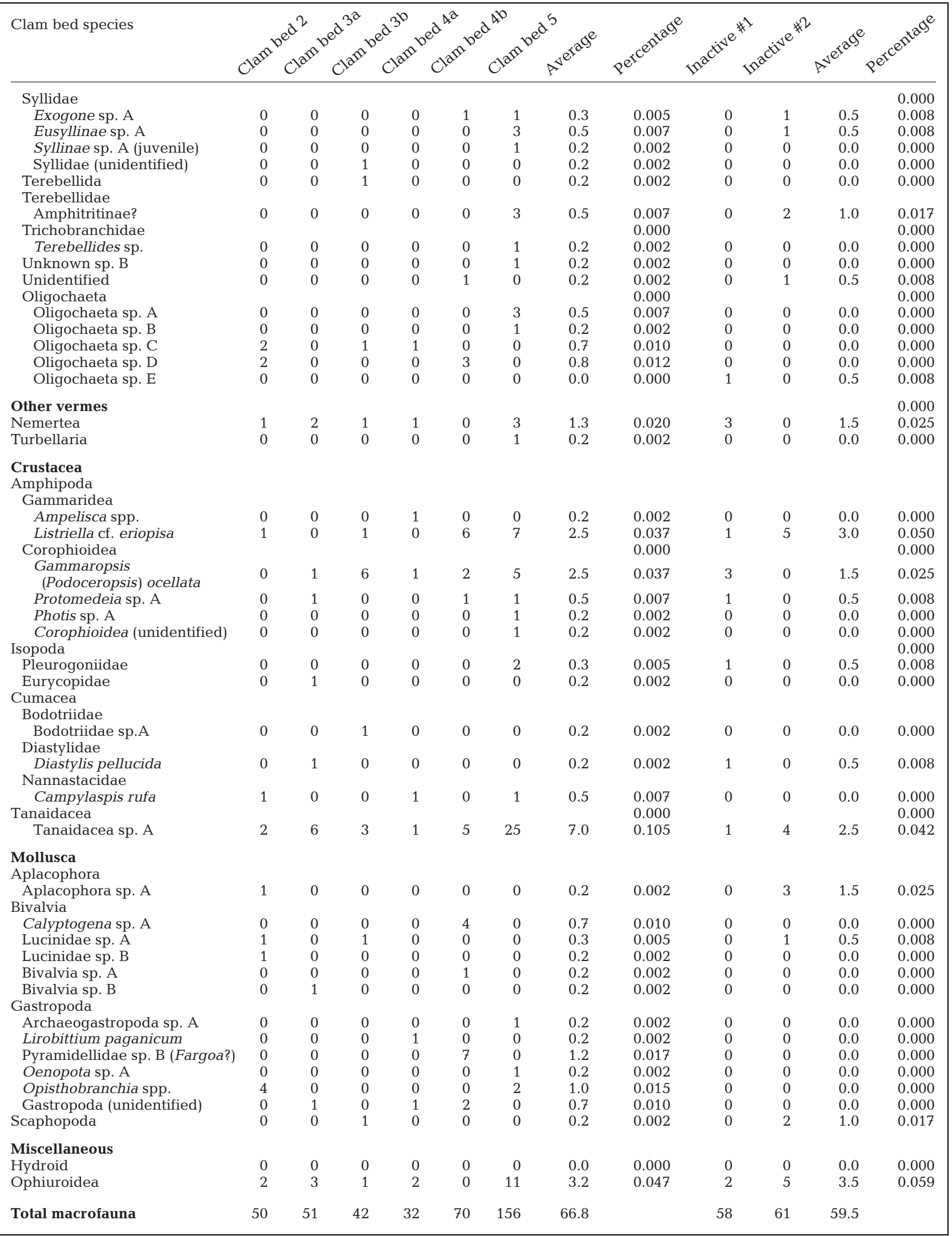


Table 6. Density and biomass of macrofauna $(>0.3 \mathrm{~mm})$ in seep and inactive sediments on the northern California margin $(500 \mathrm{~m}$ deep). Calyptogena pacifica are excluded. a and b: separate cores from the same clam bed

\begin{tabular}{|c|c|c|c|c|c|c|c|c|c|c|c|c|}
\hline \multirow[b]{2}{*}{ Clam bed: } & \multirow[b]{2}{*}{ Bed 2} & \multirow[b]{2}{*}{ Bed 3a } & \multirow[b]{2}{*}{ Bed 3b } & \multicolumn{2}{|c|}{ Seep } & \multirow[b]{2}{*}{ Bed 5} & \multirow[b]{2}{*}{ Mean } & \multirow[b]{2}{*}{ SE } & \multirow{2}{*}{ Site 1} & \multirow{2}{*}{$\begin{array}{l}\text { Inac } \\
\text { Site } 2\end{array}$} & \multirow{2}{*}{$\begin{array}{l}\text { ive } \\
\text { Mean }\end{array}$} & \multirow[b]{2}{*}{$\mathrm{SE}$} \\
\hline & & & & Bed 4a & Bed 4b & & & & & & & \\
\hline Ind. $\mathrm{m}^{-2}$ & 9365 & 9552 & 7867 & 5994 & 13111 & 29219 & 12518 & 3475 & 10863 & 11425 & 11444 & 140.48 \\
\hline \multicolumn{13}{|l|}{ Biomass, $\mathrm{g} \mathrm{m}^{-2}$} \\
\hline Annelida & 3.36 & 7.83 & 5.82 & 4.32 & 8.25 & 37.91 & 11.25 & 5.39 & 8.95 & 57.40 & 33.18 & 12.11 \\
\hline Other & 2.95 & 2.07 & 3.88 & 17.81 & 3.13 & 45.51 & 12.56 & 7.02 & 1.51 & 0.90 & 1.20 & 0.15 \\
\hline Total & 6.31 & 9.90 & 9.70 & 22.13 & 11.38 & 83.43 & 23.81 & 12.12 & 10.47 & 58.29 & 34.38 & 11.96 \\
\hline
\end{tabular}

consistent with a marine phytoplankton-based food chain. There was little evidence of lighter $\delta^{13} \mathrm{C}$ signatures that would indicate chemosynthetically derived food sources in shelf sediments. However, some of the capitellids, Mediomastus, Heteromastus and Capitella sp., and polychaetes in the genera Nephtys and Sternaspis, exhibited lower $\delta^{13} \mathrm{C}$ values $(-19.2$ to -23.0$)$ than other infauna (Table 8$). \delta^{15} \mathrm{~N}$ values were quite variable, probably reflecting a range of trophic levels, but did not differ among shelf sites.

Of the 19 infaunal invertebrates examined isotopically from clam beds on the slope, those from clam bed 1 exhibited lighter $\delta^{13} \mathrm{C}$ signatures $(-24.6 \pm 1.1)$ (Fig. 5) than those for individuals from clam bed 2 $(-19.9 \pm 0.3)$, or inactive sediments $(-18.6 \pm 0.6)\left(F_{2,25}\right)$ $=12.93, \mathrm{p}<0.001)$. Macrofauna from clam beds 1 and 2 had lighter average $\delta^{13} \mathrm{C}$ signatures than those from shelf sites $\left(F_{3,95}=43.3, \mathrm{p}<0.001\right)$. A single dorvilleid polychaete, Dorvillea sp., had a very light $\delta^{13} \mathrm{C}$ signature (-33.5), suggestive of chemoautotrophic symbionts or selective feeding on free-living chemoautotrophic bacteria. $\delta^{15} \mathrm{~N}$ values were variable (Fig. 5), but did not differ among clam beds and inactive sediments. However, $\delta^{15} \mathrm{~N}$ values were considerably greater for slope macrofauna from the 2 clam beds and inactive slope sediments (Fig. 5) than for shelf macrofauna (Table 8) $\left(F_{3,95}=25.2, \mathrm{p}<0.001\right)$.

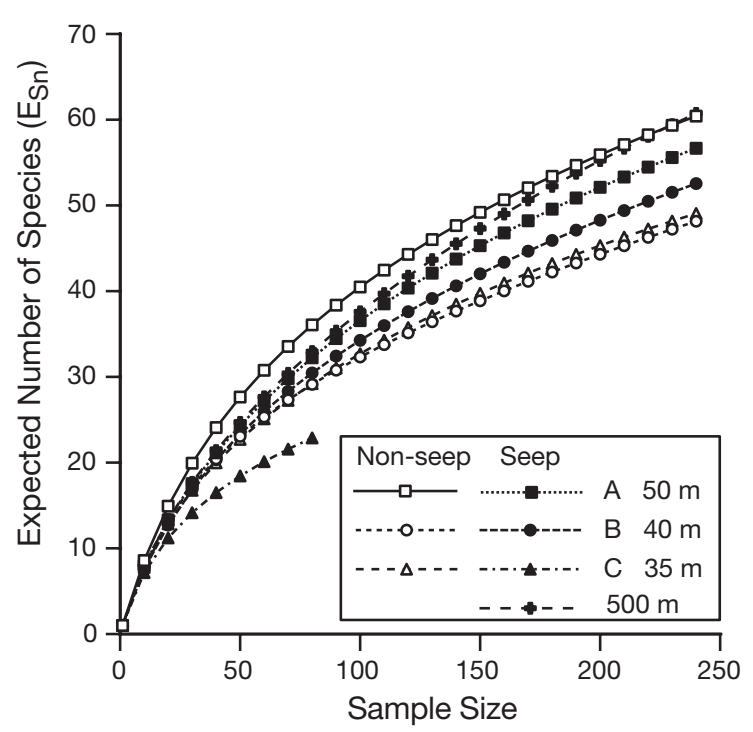

Fig. 4. Rarefaction curves showing expected number of macrofaunal species $(\geq 0.3 \mathrm{~mm}$ ) for a given sample size (no. of ind.) in pooled samples (seep vs non-seep) from 4 locations on the northern California margin

Table 7. Diversity indices of shelf and slope macrofauna on the northern California margin. Data are for pooled cores unless otherwise indicated. ${ }^{* *}$ Average of 6 cores

\begin{tabular}{|c|c|c|c|c|c|c|c|c|c|}
\hline \multirow[b]{2}{*}{ Information index $H^{\prime}\left(\log _{2}\right)$} & \multicolumn{2}{|c|}{$\begin{array}{c}\text { Site A } \\
\text { Seep Non-seep }\end{array}$} & \multicolumn{2}{|c|}{$\begin{array}{c}\text { Site B } \\
\text { Seep Non-seep }\end{array}$} & \multicolumn{2}{|c|}{$\begin{array}{c}\text { Site C } \\
\text { Seep Non-seep }\end{array}$} & \multicolumn{2}{|c|}{$\begin{array}{cc} & \text { Slope } \\
\text { Seep } & \text { Inactive site 1 }\end{array}$} & \multirow{2}{*}{$\begin{array}{c}\text { Inactive site } 2 \\
3.89\end{array}$} \\
\hline & 5.02 & 5.22 & 4.66 & 4.80 & 3.85 & 4.76 & $\begin{array}{c}4.95 \\
(3.83)^{*}\end{array}$ & 3.45 & \\
\hline Evenness $J^{\prime}$ & 0.76 & 0.87 & 0.77 & 0.79 & 0.85 & 0.76 & $\begin{array}{c}0.79 \\
(0.85)^{*}\end{array}$ & 0.81 & 0.85 \\
\hline Rank 1 dominance (\%) & $14.4^{\mathrm{a}}$ & $10.5^{\mathrm{b}}$ & $24.0^{\mathrm{c}}$ & $14.7^{\mathrm{c}}$ & $18.5^{\mathrm{d}}$ & $18.7^{\mathrm{d}}$ & $17.7^{\mathrm{e}}$ & $27.6^{\mathrm{f}}$ & $13.1^{\mathrm{e}}$ \\
\hline Most abundant species: & \multicolumn{3}{|c|}{$\begin{array}{l}\text { a Lumbrineris sp. } \\
{ }^{\mathrm{b}} \text { Chaetozone hartmanae } \\
{ }^{\mathrm{c}} \text { Tecticeps convexus }\end{array}$} & \multicolumn{3}{|c|}{$\begin{array}{l}\mathrm{d} \text { Nephtys californiensis } \\
{ }^{\mathrm{e}} \text { Mediomastus californiensis } \\
{ }^{\mathrm{f}} \text { Levinsenia gracilis }\end{array}$} & & & \\
\hline
\end{tabular}


Table 8. Stable isotopic signatures of northern California shelf macrofauna

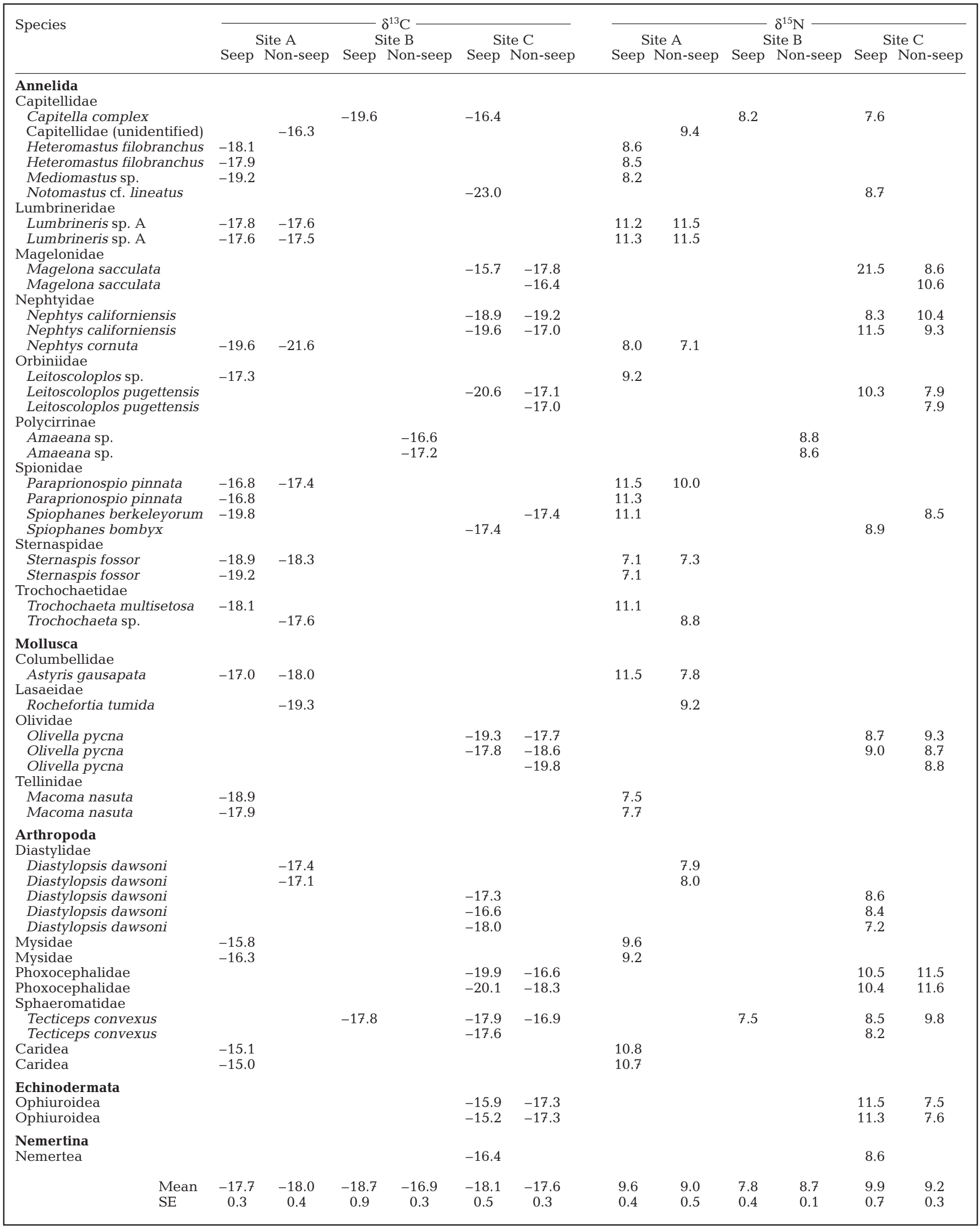




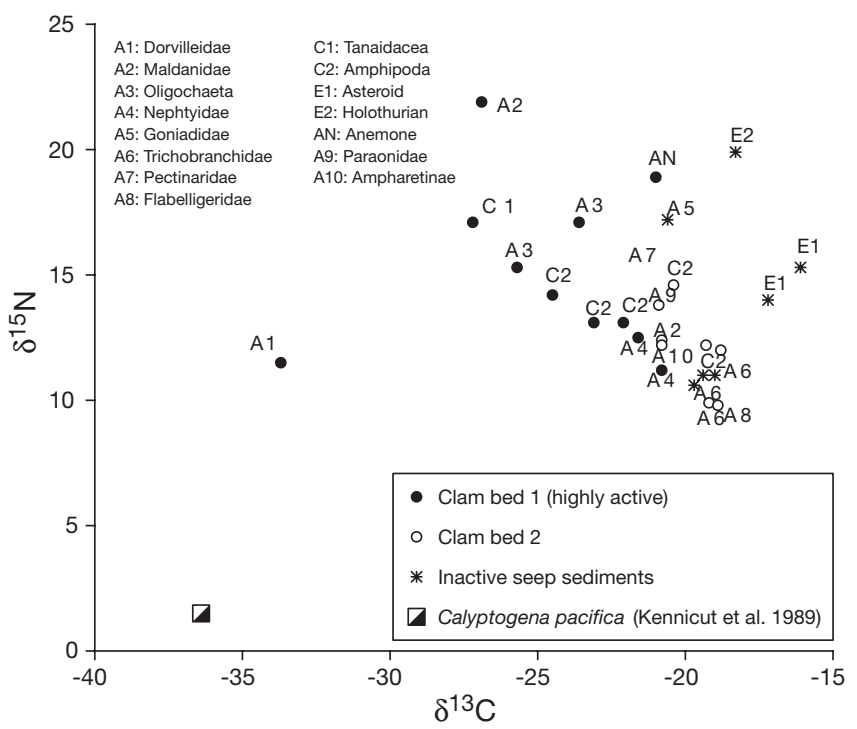

Fig. 5. Dual isotope plot of infaunal invertebrate isotopic signatures from 3 locations at ca $500 \mathrm{~m}$ deep on the northern California margin. $\mathrm{A}=$ annelid, $\mathrm{C}=$ crustacean, $\mathrm{E}=$ echinoderm

\section{DISCUSSION}

\section{Is there evidence for specialization among seep macrofauna?}

\section{Shelf}

The few existing studies comparing seep to non-seep macrofauna have yielded varied results that differ with setting and taxon (Table 1). At shelf depths, hydrocarbon seeps (Davis \& Spies 1980, Montagna et al. 1989) methane pockmarks (Dando et al. 1991, 1994), and 'bubbling reef' sediments (Jensen et al. 1992) support many taxa typical of non-seep settings. Some seep specialists with chemosynthetic symbionts are present in these environments, but they are usually megafaunal (pogonophorans and thyasurid bivalves) or meiofaunal (nematodes). The northern California shelf and slope faunas characterized in this study do not appear to differ substantially from nearby non-seep assemblages. The most common infaunal taxa were present in both seep and non-seep sediments. Our data suggest that some rarer forms may select for or against seep settings on the shelf (Table 4), but confirmation of habitat preferences would require additional collections. Like Vanreusel et al.'s (1997) multivariate comparisons of hydrothermal vent and non-vent nematode genera, we found greater similarity of macrofaunal communities in seep and non-seep sediments compared within shelf sites (A, B, or C) than among seep faunas at different sites (Fig. 2, Table 4). We speculate that shelf environmental properties such as flow, particle size or localized terrestrial inputs can have greater influence on infaunal community composition than methane seepage.

The regular presence of Capitella sp. in shelf seep sediments, and their absence in our non-seep samples, is consistent with known sulfide tolerances for the genus (Thiermann et al. 1997, Gamenick et al. 1998a,b). Capitella may cue to sulfides during settlement (Cuomo 1985) or may require sulfidic conditions for maximal growth (Tsutsumi 1997). However, Capitella never comprised more than $5 \%$ of the shelf seep assemblage, and was absent in seeps on the slope (Table 4). Amphipods in the genus Rhepoxynius are sensitive to sulfides (Knezovich et al. 1996). Their apparent avoidance of seeps on the northern California shelf is consistent with this behavior. At Site A, the most quiescent and fine-grained of the shelf sites, the dominant taxa Lumbrineris sp., Mediomastus spp., and Nephtys cornuta together comprised $>41 \%$ of the seep fauna but only $13 \%$ of the non-seep fauna. These deposit-feeding taxa, capable of responding to sediment enrichment, contribute to higher dominance in seep than non-seep sediments.

\section{Slope}

The infaunal assemblage at the $500 \mathrm{~m}$ seep site was generally characteristic of North American upper slope sediments (Lissner et al. 1988, Hyland et al. 1991). Exceptions were the vesicomyid Calyptogena pacifica and lucinid bivalves, which are symbiont-bearing and characteristic of seep or oxygen minimum zone sediments (Sibuet \& Olu 1998, Levin et al. 2000). Together the polychaetes Mediomastus spp. and Levinsenia gracilis comprised over $25 \%$ of the infauna. Mediomastus spp. are typical of non-seep sediments, but usually occur at shallower depths than $500 \mathrm{~m}$ (Lissner et al. 1988). Smith et al. (1998) studied infaunal community composition beneath and near another deep-water reducing environment, at whale falls in the Santa Catalina Basin (1240 m). As in the present study, they found little evidence of specialization on enrichment or sulfides.

Quantitative macrofaunal density estimates were available in the present study only for slope seep sediments. The average macrofaunal density at the $500 \mathrm{~m}$ seep (12518 ind. $\mathrm{m}^{-2}$ ), was typical of bathyal margins of the Atlantic (Blake \& Grassle 1994), Pacific (Lissner et al. 1988, Hyland et al. 1991, Vetter \& Dayton 1998) and Indian Oceans (Levin et al. 1997, 2000). Density differences among cores from different northern California clam beds could reflect differing levels of seepage in each bed. Clam bed 5, with high densities of 
Mediomastus spp. and dorvilleids, appeared more active than the other clam beds. Unfortunately, no pore-water profiles were available to confirm this. Other investigators have reported elevated densities of meiofauna (Buck \& Barry 1998) and macrofauna (Davis \& Spies 1980) at seeps.

Reduced macrofaunal diversity at methane seeps might be expected if sediments were physiologically stressful or were organically enriched settings (Levin \& Gage 1998). Reduced macrofaunal diversity has been reported from North Sea pockmarks (115 m) (Dando et al. 1991) and from hydrothermal settings in the Galapagos, at $21^{\circ} \mathrm{N}$ (2600 to $2700 \mathrm{~m}$, Grassle et al. 1985) and in the Aegean Sea (5 to $10 \mathrm{~m}$, Thiermann et al. 1997). Vanreusel et al. (1997) noted reduced species diversity and greater dominance in nematode assemblages at hydrothermal vents in the North Fiji Basin. We did not observe any diversity reduction in most northern California shelf seep patches, except at Site C (35 m) (Fig. 4, Table 7). Hydrocarbon seeps off Santa Barbara $(16 \mathrm{~m})$ also did not exhibit reduced macrofaunal diversity (Montagna et al. 1989).

\section{Do seep infauna exhibit nutritional specialization?}

Food sources for the shelf and slope infauna on the northern California margin may include phytoplankton-based organic matter, terrestrial material deposited by Eel River flood events, and chemosynthetically derived organic matter. Marine phytoplankton in productive coastal waters typically have $\delta^{13} \mathrm{C}$ signatures between -15 and -23 at temperate latitudes (Fry \& Wainright 1991) and approximately -19 on the Eel River slope (Leithold \& Hope 1999). Organic matter from the Eel River has a $\delta^{13} \mathrm{C}$ value of about -25 (Leithold \& Hope 1999), whereas invertebrates with symbiotic sulfur oxidizing or methane consuming bacteria may have $\delta^{13} \mathrm{C}$ signatures of -30 to -70 (Brooks et al. 1987, Conway et al. 1994). Based on average $\delta^{13} \mathrm{C}$ values, which are between -17 and -19 (Table 8), shelf faunas of both seep and non-seep sediments appear to rely primarily on phytoplankton-based organic matter. This occurs despite the fact that terrestrial debris (twigs and wood chips) was visible in most sediment samples.

The absence of strong chemosynthetic contribution to nutrition of benthos appears to be a widespread feature of shallow water seeps and vents. Carbon isotopic studies of macrofauna in a North Sea methane seep revealed most fauna with $\delta^{13} \mathrm{C}$ signatures between -16 and -20 , indicating little nutritional contribution of methane-derived carbon or sulfur oxidation (Dando et al. 1991). Similar results were obtained for epifauna on carbonates produced at seeps in the Kattegat (10 to
$12 \mathrm{~m}$, Jensen et al. 1992). Tissues of benthic fishes and crustaceans at a $132 \mathrm{~m}$ methane seep off Oregon exhibited detectable but very minor contributions of seep-associated C and N (Juhl \& Taghon 1993). The lesser dependence on chemoautotrophic-based food sources exhibited by shallow-water seep macrofauna, relative to those at deep-water seeps (Conway et al. 1994), may result from the greater availability of photosynthetically produced food in shallow water.

On the Eel River margin, isotopic signatures of the slope fauna (Fig. 5) differ from those on the shelf (Table 8). The lighter $\delta^{13} \mathrm{C}$ and higher $\delta^{15} \mathrm{~N}$ values for the slope organisms suggest one or more of the following scenarios: (1) organics derived from Eel River flood deposits may be a significant dietary component of infauna, (2) sulfur oxidizing or methanotrophic bacteria may be of nutritional importance in combination with a phytoplankton-based diet, and (3) the organic matter is derived from heavy, upwelled nitrogen, or is extensively reworked before reaching the slope, creating high $\delta^{15} \mathrm{~N}$ values. Approximately $5 \%$ of the annual Eel River sediment discharge accumulates on the slope between 450 and 600 m (Alexander \& Simoneau 1999), providing a ready source of lighter carbon and recycled nitrogen. Only a dorvilleid polychaete and presumably the clam Calyptogena pacifica (analyzed from this region by Kennicutt et al. 1989) exhibited the light $\delta^{13} \mathrm{C}$ values characteristic of chemosynthesis-based nutrition. However, the lighter $\delta^{13} \mathrm{C}$ of clam bed 1 infauna (Fig. 5) may indicate some reliance by other taxa on chemosynthetic bacteria. Overall, the slope observations suggest that there are nutritional differences among species, among seep sites (clam beds) on scales of 10 s of meters, and between inactive and active sites.

\section{Conclusions}

Information about macrofaunal composition, density, diversity and trophic pathways suggests that methane seeps on the northern California continental shelf and slope do not support macrofaunal assemblages that are highly distinct from the ambient faunas. On the shelf, seep patches are small and may be ephemeral due to frequent, storm-induced disturbance and shifting of methane vent sites. Shelf seeps appear to be inhabited by a subset of the ambient shelf fauna that is tolerant of sulfidic conditions. The absence of typical seep epifauna (vesicomyid clams, vestimentiferan or pogonophoran tubeworms, and bacterial mats) suggests that the shelf seep habitats sampled were too small or short lived for trophic specialization to occur among infauna. In addition, the high mobility of many shelf organisms, particularly the crustaceans, might reduce the likelihood that they will remain localized within seep 
patches. Our sampling took place just following a major storm that resuspended bottom sediments on the shelf. Bacterial mats have been observed at Site B during summer by other investigators (Orange et al. 1997, Orange et al. pers. comm.).

The slope seep macrofauna exhibited typical upper bathyal densities, taxonomic composition, and diversity patterns. A similar result has been obtained for foraminifera sampled at our same slope study site (Rathburn et al. 2000). The seeps support several macrofaunal species that utilize either symbiotic or free-living chemosynthetic bacteria. However, the majority of infaunal taxa are carnivores or deposit feeders that probably utilize mainly terrestrial or marine organic matter of photosynthetic origin.

It would be premature to generalize these findings to other seep environments. Shelf and shallow bathyal faunas regularly encounter patches of disturbed, stressed or sulfidic sediments in non-seep circumstances, such as beneath large-animal or plant falls (Stockton \& DeLaca 1982, Smith et al. 1998) or within oxygen minimum zones (Gallardo et al. 1995, Levin \& Gage 1998). Thus, at least some bathyal and shelf taxa may be preadapted to the geochemical conditions associated with seeps. Abyssal faunas, however, may be less likely to experience sulfidic or hypoxic conditions in the absence of seepage or venting. As a result, we predict that physiological, trophic, and morphological specialization, as well as shifts in community structure, are potentially more likely among seep infauna on the continental rise and abyss.

Acknowledgements. We thank the captains and crews of the RV 'Laney Chouest' and the RV 'McGaw', the pilots and staff of the 'Delta' and 'Sea Cliff' submersibles and ROV 'Scorpio'. For assistance at sea we thank M. Tryon, K. Brown, M. Saladin, Z. Held, J. Gieskes, C. Mahn, A. Baco, and N. von Mirbach. Non-polychaete identifications were provided by D. Cadien. Oxygen analyses were run by R. Patrick and D. Masten at the Norpax facility at the Scripps Institution of Oceanography. Comments from anonymous reviewers improved the manuscript. The research was supported by Grants UAF 97-0037, 98-0038, and 00-0050 from the NOAA National Undersea Research Program, Alaska, and by matching funds from the Scripps Institution of Oceanography Director's office.

\section{LITERATURE CITED}

Alexander CR, Simoneau AM (1999) Spatial variability in sedimentary processes on the Eel continental slope. Mar Geol 154:243-254

Barry JP, Greene HG, Orange DL, Baxter CH, Robison BH, Kochevar RE, Nybakken JW, Reed DL, McHugh CM (1996) Biologic and geologic characteristics of cold seeps in Monterey Bay, California. Deep-Sea Res 43:1739-1762

Barry JP, Kochevar RE, Baxter CH (1997) The influence of pore-water chemistry and physiology in the distribution of vesicomyid clams at cold seeps in Monterey Bay: implications for patterns of chemosynthetic community organization. Limnol Oceanogr 42:318-328

Beauchamp B, Krouse HR, Harrison JC, Nassichuk WW, Eliuk LS (1989) Cretaceous cold-seep communities and methane-derived carbonates in the Canadian Arctic. Science 244:53-55

Blake JA, Grassle JF (1994) Benthic community structure on the U.S. South Atlantic slope off the Carolinas: spatial heterogeneity in a current-dominated system. Deep-Sea Res II 41:835-874

Blake JA, Scott PV (1997) Taxonomic atlas of the Santa Maria Basin and western Santa Barbara Channel, Vol 1. Santa Barbara Museum of Natural History, Santa Barbara, CA

Boulegue J, Benedetti EL, Dron D, Mariotti A, Letolle R (1987a) Geochemical and biogeochemical observations on the biological communities associated with fluid venting in Nankai Trough and Japan Trench subduction zones. Earth Planet Sci Lett 83:343-355

Boulegue J, Iijama JT, Charlou JL, Jebwab J (1987b) Nankai Trough, Japan Trench and Kuril Trench: geochemistry of fluids sampled by submersible 'Nautile'. Earth Planet Sci Lett 83:363-375

Brewer PG, Orange D, Friederich G, Peltzer E, Kvenvolden KA, Lorenson T, Bullister JL, Orr F Jr (1997) Gas hydrates and global change: a preliminary case study offshore northern California. EOS Transactions. AGU 78 (46):F340

Brooks JM, Kennicutt MC II, Fisher CR, Macko SA, Cole K, Childress JJ, Bidigare RR, Vetter RD (1987) Deep-sea hydrocarbon seep communities: evidence for energy and nutritional carbon sources. Science 238:1138-1142

Buck KR, Barry JP (1998) Monterey Bay cold seep infauna: quantitative comparison of bacterial mat meiofauna with non-seep control sites. Cah Biol Mar 39:333-335

Cary C, Fry B, Felbeck H, Vetter RD (1989) Multiple trophic resources for a chemoautotrophic community at a cold water brine seep at the base of the Florida Escarpment. Mar Biol 100:411-418

Clarke KR, Warwick RM (1994) Change in marine communities: an approach to statistical analysis and interpretation. Natural Environmental Research Council, United Kingdom and Plymouth Marine Laboratory, Plymouth

Conway N, Kennicutt M II, Van Dover C (1994) Stable isotopes in the study of marine chemosynthetic based ecosystems. In: Lajtha K, Michener R (eds) Stable isotopes in ecology and environmental sciences. Blackwell Scientific Publications, London, p 158-186

Cuomo MC (1985) Sulphide as a larval settlement cue for Capitella sp. I. Biogeochemistry 1:169-181

Dando PR, Austen MC, Burke RA Jr, Judd AG, Moore DC, O'Hara SCM, Schmaljohann R, Southward AJ (1991) Ecology of a North Sea pockmark with an active methane seep. Mar Ecol Prog Ser 70:49-63

Dando PR, Bussmann I, Niven SJ, O'Hara SCM, Schmaljohann R, Taylor LJ (1994) A methane seep area in the Skagerrak, the habitat of the pogonophore Siboglinum poseidoni and the bivalve mollusc Thyasira sarsi. Mar Ecol Prog Ser 107:157-167

Davis PH, Spiess RB (1980) Infaunal benthos of a natural petroleum seep: study of community structure. Mar Biol 59:31-41

Dron D, Boulegue J, Taira A, Rangin C (1987) Geochemistry of the Tenryu Canyon deep sea fan biological community (Kaiko). Earth Planet Sci Lett 83:356-362

Fry B, Wainright SC (1991) Diatom sources of ${ }^{13} \mathrm{C}$-rich carbon in marine food webs. Mar Ecol Prog Ser 76:149-157

Gallardo VA, Carrasco FD, Roa R, Canete JJ (1995) Ecological 
patterns in the benthic macrobiota across the continental shelf off central Chile. Ophelia 40:167-188

Gamenick I, Abbiati M, Giere O (1998a) Field distribution and sulphide tolerance of Capitella capitata (Annelida: Polychaeta) around shallow water hydrothermal vents off Milos (Aegean Sea). A new sibling species? Mar Biol 130: 447-453

Gamenick I, Vismann B, Grieshaber MK, Giere O (1998b) Ecophysiological differentiation of Capitella capitata (Polychaeta). Sibling species from different sulfidic habitats. Mar Ecol Prog Ser 175:155-166

Gamo T, Sakai H, Ishibashi JI, Shitashima K, Boulegue J (1992) Methane, ethane and total inorganic carbon in fluid samples taken during the 1989 Kaiko-Nankai Project. Earth Planet Sci Lett 109:383-390

Grassle JF, Brown-Leger S, Morese-Porteous L, Petrecca R, Williams I (1985) Deep-sea fauna of sediments in the vicinity of hydrothermal vents. Biol Soc Wash Bull 6:443-452

Guezennec J, Fiala-Medioni A (1996) Bacterial abundance and diversity in the Barbados Trench determined by phospholipid analysis. FEMS Microbiol Ecol 19:83-93

Han MW, Suess E (1986) Subduction-induced pore fluid venting and the formation of authigenic carbonates along the Cascadia continental margin: implications for the global Ca-cycle. Palaeogeogr Palaeoclimatol Palaeoecol 71:97-118

Hovland M, Judd AG (1988) Seabed pockmarks and seepages, impact on geology, biology and the marine environment. Graham and Trotman Inc Publ, London

Hurlbert S (1971) The nonconcept of species diversity: a critique and alternative parameters. Ecology 52:577-586

Hyland J, Baptiste E, Campbell J, Kennedy J, Kropp R, Williams S (1991) Macroinfaunal communities of the Santa Maria Basin on the California outer continental shelf and slope. Mar Ecol Prog Ser 78:147-161

Jensen P (1986) Nematode fauna in the sulphide-rich brine seep and adjacent bottoms of the East Flower Garden, NW Gulf of Mexico. Mar Biol 92:489-503

Jensen P, Aagaard I, Burke R Jr, Dando P, Jorgensen N, Kuijpers A, Laier T, O'Hara S, Schmaljohann R (1992) 'Bubbling reefs' in the Kattegat: submarine landscapes of carbonate-cemented rocks support a diverse ecosystem at methane seeps. Mar Ecol Prog Ser 83:103-112

Jones RW (1993) Preliminary observations on benthonic foraminifera associated with biogenic gas seep in the North Sea. In: Jenkins DG (ed) Applied micropaleontology. Kluwer Academic Publishers, Dordrecht, p 69-91

Jones RW (1996) Preliminary observations on benthonic foraminifera associated with petroleum seeps. In: Jones RW (ed) Micropaleontology in petroleum exploration. Oxford University Press, New York, p 179-196

Juhl A, Taghon G (1993) Biology of an active methane seep on the Oregon continental shelf. Mar Ecol Prog Ser 102: 287-294

Juniper SK, Sibuet M (1987) Cold seep benthic communities in Japan subduction zones; spatial organization, trophic strategies and evidence for temporal evolution. Mar Ecol Prog Ser 40:115-126

Kennicutt MC II, Brooks JM, Bidigare RR, McDonald SJ, Adkison DL, Macko SA (1989) An upper slope 'cold' seep community: northern California. Limnol Oceanogr 34: $635-640$

Knezovich JP, Steichen DJ, Jelinski JA, Anderson SL (1996) Sulfide tolerance of four marine species used to evaluate sediment and pore-water toxicity. Bull Environ Contam Toxicol 57:450-457

Kulm LD, Suess E, Moore JC, Carson B, Lewis BT, Ritger
SD, Kadko DC, Thornburg TM, Embley RW, Rugh WD, Massoth GJ, Langseth MG, Cochrane GR, Scamman RL (1986) Oregon subduction zone: venting, fauna and carbonates. Science 231:561-566

Leithold EL, Hope RS (1999) Deposition and modification of a flood layer on the northern California shelf: lessons from and about the fate of terrestrial particulate organic carbon. Mar Geol 154:183-195

Levin LA, Gage JD (1998) Relationships between oxygen, organic matter and the diversity of bathyal macrofauna. Deep-Sea Res II 45:129-164

Levin LA, Gage J, Lamont P, Cammidge L, Patience A, Martin $C$ (1997) Infaunal community structure in a low-oxygen organic rich habitat on the Oman margin. In: Hawkins L, Hutchinson S, Jenson A, Williams J, Sheader M (eds) Responses of marine organisms to their environment. 30th Eur Mar Biol Symp. Southampton Oceanography Centre, Southampton, p 223-230

Levin LA, Gage JD, Martin C, Lamont PA (2000) Macrobenthic community structure within and beneath the oxygen minimum zone, NW Arabian Sea. Deep-Sea Res II: 47: $189-226$

Lissner A, Diener D, Kanter R (1988) Biological reconnaissance of selected benthic habitats within three California OCS planning areas. Data report, November 1988. SAIC and MEC Analytical Systems, Inc, MMS contract no. 14-12-0001-30388

MacDonald IR, Guinasso J, Reilly JF, Brooks JM, Callender WR, Gabrielle SG (1990) Gulf of Mexico hydrocarbon seep communities: VI. Patterns of community structure and habitat. Geo-Mar Lett 10:244-252

Masuzawa T, Kitagawa H, Kusakabe M (1992) Sulfate reduction using methane in sediments beneath a bathyal cold seep giant clam community off Hatsushima Island, Sagami Bay, Japan. Earth Planet Sci Lett 110:39-50

McAleece N, Lambshead J, Paterson G, Gage J, Harris P, Lamont P (1997) BioDiversity Pro. The Natural History Museum and the Scottish Association For Marine Science, London

McCall PL, Tevesz MJS (1982) Animal-sediment relations. The biogenic alteration of sediments. Plenum Press, New York

Miura T, Hashimoto J (1991) Nicomache ohtai, new species (Polychaeta: Maldanidae) collected from the Hatsushima Cold-Seep in Sagami Bay. Proc Biol Soc Wash 104: 159-165

Montagna PA, Bauer JE, Hardin D, Spies RB (1989) Vertical distribution of microbial and meiofaunal populations in sediments of a natural coastal hydrocarbon seep. J Mar Res 47:657-680

Olu K, Duperret A, Sibuet M, Foucher JP, Fiala-Medioni A (1996a) Structure and distribution of cold seep communities along the Peruvian active margin: relationship to geological and fluid patterns. Mar Ecol Prog Ser 132:109-125

Olu K, Sibuet M, Harmegnies F, Foucher JP, Fiala-Medioni A (1996b) Spatial distribution of diverse cold seep communities living on various diapiric structures of the southern Barbados prism. Prog Oceanogr 38:347-376

Olu K, Lance S, Sibuet M, Henry P, Fiala-Medioni A, Dinet A (1997) Cold seep communities as indicators of fluid expulsion patterns through mud volcanoes seaward of the Barbados Accretionary Prism. Deep-Sea Res 44:811-841

Orange DL (1997) Active and episodic fluid flow on the southern Cascadia continental shelf and slope. EOS Transactions, AGU 78(46):F667

Paull CK, Hecker B, Commeau R, Freeman-Lynde RP, Neumann C, Corso WP, Golubic S, Hook JE, Sikes E, Curray J 
(1984) Biological communities at the Florida Escarpment resemble hydrothermal vent taxa. Science 226:965-967

Paull CK, Jull AJT, Tolin LJ, Linick T (1985) Stable isotope evidence for chemoautotrophy in an abyssal seep community. Nature 317:709-711

Petrecca RF, Grassle JF (1987) Notes on fauna from several deep-sea hydrothermal vent and cold seep soft-sediment communities. In: McMurray GR (ed) Gorda Ridge, a seafloor spreading center in the United States' exclusive economic zone. Springer-Verlag, New York, p 279-283

Powell EN, Bright TJ (1981) A thiobios does exist-Gnathostomulid domination of the canyon community at the East Flower Garden Brine Seep. Int Rev Ges Hydrobiol 66: 675-683

Powell EN, Bright TJ, Woods A, Gittings S (1983) Meiofauna and the thiobios in the East Flower Garden Brine Seep. Mar Biol 73:269-283

Rathburn AE, Levin LA, Held Z, Lohmann KC (2000) Benthic foraminifera associated with cold methane seeps on the northern California margin: ecology and stable isotopic composition. Mar Micropaleontol 38:247-266

Schmaljohann R, Faber E, Whiticar MJ, Dando PR (1990) Coexistence of methane- and sulphur-based endosymbioses between bacteria and invertebrates at a site in the Skagerrak. Mar Ecol Prog Ser 61:119-124

Sen Gupta BK, Aharon P (1994) Benthic foraminifera of bathyal hydrocarbon vents of the Gulf of Mexico: initial report on communities and stable isotopes. Geo Mar Lett 14:88-96

Shirayama Y, Ohta S (1990) Meiofauna in a cold-seep community off Hatsushima, central Japan. J Oceanogr Soc Jpn 46:118-124

Sibuet M, Olu K (1998) Biogeography, biodiversity and fluid dependence of deep-sea cold-seep communities at active and passive margins. Deep-Sea Res II 45:517-567

Sibuet M, Juniper SK, Pautot G (1988) Cold-seep benthic communities in the Japan subduction zones: geological control of community development. J Mar Res v.46, n.2, 1988:333-348

Smith CR, Maybaum HL, Baco AR, Pope RH, Carpenter SD, Yager PL, Macko SA, Deming JW (1998) Sediment community structure around a whale skeleton in the deep Northeast Pacific: macrofaunal, microbial and bioturbation effects. Deep-Sea Res II 45:335-364

Editorial responsibility: Otto Kinne (Editor), Oldendorf/Luhe, Germany
Stockton WL, DeLaca TE (1982) Food falls in the deep sea: occurrence, quality, and significance. Deep-Sea Res 29: $157-169$

Suess E, Whiticar MJ (1989) Methane-derived $\mathrm{CO}_{2}$ in pore water fluids expelled from the Oregon subduction zone. Palaeogeogr Palaeoclimatol Palaeoecol 71:119-136

Suess E, Bohrman G, von Huene R, Linke P, Wallmann $\mathrm{K}$, Lammers S, Sahling H (1998) Fluid venting in the eastern Aleutian subduction zone. J Geophys Res 103:2597-2614

Thiermann F, Windoffer R, Giere O (1994) Selected meiofauna around shallow water hydrothermal vents off Milos (Greece): ecological and ultrastructural aspects. Vie Milieu $44: 215-226$

Thiermann F, Akoumianaki I, Huges J, Giere O (1997) Benthic fauna of a shallow-water gaseohydrothermal vent area in the Aegean Sea (Milos, Greece). Mar Biol 128:149-159

Tsutsumi H, Wainright S, Montani S, Saga M, Ichihara S (1997) Hydrogen sulfide promotes growth of the polychaete, Capitella sp. I. Benthic Ecology Meeting. March 1997. Rutgers, NJ (Abstract)

Van Dover CL, Fry B (1989) Stable isotopic compositions of hydrothermal vent organisms. Mar Biol 102:257-263

Vanreusel A, Van den Bossche I, Thiermann F (1997) Freeliving marine nematodes from hydrothermal sediments: similarities with communities from diverse reduced habitats. Mar Ecol Prog Ser 157:207-219

Vetter EW, Dayton PK (1998) Macrofaunal communities within and adjacent to a detritus-rich submarine canyon system. Deep-Sea Res II 45:25-54

Wade TL, Kennicutt MC, Brooks JM (1989) Gulf of Mexico hydrocarbon seep communities: Part III. Aromatic hydrocarbon concentrations in organisms, sediments and water. Mar Environ Res 27:19-30

Yun JW, Orange DL, Field ME (1999) Subsurface gas offshore of northern California and its link to submarine geomorphology. Mar Geol 154:357-368

Zuleger E, Gieskes JM, You CF (1995) Sediment cores on the Costa Rica Accretionary Prism (Atlantis II Cruise 131-10): pore water studies. Scripps Inst Oceanogr Ref Ser 95-4: p 75

Zuleger E, Gieskes JM, You CF (1996) Interstitial water chemistry of sediments of the Costa Rica accretionary complex off the Nicoya Peninsula. Geophys Res Lett 23:899-902

Submitted: January 14, 2000; Accepted: October 13, 2000

Proofs received from author(s): November 29, 2000 